\title{
Mesures quasi-Bernoulli au sens faible : résultats et exemples
}

\author{
Benoît Testud \\ Laboratoire de Mathématiques, Université Blaise Pascal, 24, avenue des Landais, 63177 Aubière cedex, France \\ Reçu le 8 octobre 2003 ; reçu en forme révisée le 12 juillet 2004 ; accepté le 3 janvier 2005 \\ Disponible sur Internet le 22 juin 2005
}

\section{Résumé}

On introduit une nouvelle condition d'homogénéité (quasi-Bernoulli au sens faible) sur les mesures sous laquelle le formalisme multifractal fonctionne. Cette condition englobe le cas classique où la mesure est quasi-Bernoulli et permet donc de raffiner les résultats obtenus successivement par Michon, Brown, Peyrière et Heurteaux. On met ensuite en évidence certaines mesures autosimilaires qui ne satisfont pas les conditions classiques de séparation, non quasi-Bernoulli mais qui vérifient la condition mentionnée auparavant. Ces mesures interviennent par exemple lors de l'analyse de certains graphes autoaffines déjà étudiés par McMullen, Przytycki et Urbański, Kenyon et Peres.

(c) 2005 Elsevier SAS. Tous droits réservés.

\begin{abstract}
We introduce a new homogeneity condition (weak quasi-Bernoulli) to the measures under which the multifractal formalism holds. This condition includes the well known case where the measure is quasi-Bernoulli and thus we can precise the results obtained successively by Michon, Brown, Peyrière and Heurteaux. Then, we highlight some self similar measures with overlaps which are not quasi-Bernoulli but satisfy the condition mentioned above. Moreover, we show how these measures can be used to analyse some self affine graphs studied before by McMullen, Przytycki and Urbański, Kenyon and Peres.

(c) 2005 Elsevier SAS. Tous droits réservés.

Mots-clés : Mesure quasi-Bernoulli; Mesure de Gibbs; Mesure autosimilaire; Mesure autoaffine ; Dimension de Hausdorff; Dimension de

Tricot; Analyse multifractale

Keywords: Quasi-Bernoulli measure; Gibbs measure; Self similar measure; Self affine measure; Hausdorff dimension; Tricot dimension;

Multifractal analysis; Multifractal analysis
\end{abstract}

\section{Abridged English version}

Let $\ell \geqslant 2$ be an integer and $\sigma$ be the shift map on [0,1[, that is:

Adresse e-mail : Testudbe @ math.univ-bpclermont.fr. 


$$
\begin{aligned}
\sigma:[0,1[ & \rightarrow[0,1[, \\
x & \rightarrow \ell x(\bmod 1) .
\end{aligned}
$$

We denote by $\mathcal{F}_{n}$ the set of $\ell$-adic intervals of the $n$th generation included in [0,1[. For every $x \in\left[0,1\left[, I_{n}(x)\right.\right.$ stands for the unique interval among $\mathcal{F}_{n}$ which contains $x$.

For a given probability measure $m$ on the interval $[0,1$, one goal of the multifractal analysis is to determine an explicit formula between the dimension of the sets $E_{\alpha}$ defined by

$$
\forall \alpha>0, \quad E_{\alpha}=\left\{x \in \left[0,1\left[, \lim _{n \rightarrow+\infty} \frac{\log m\left(I_{n}(x)\right)}{\log \left|I_{n}(x)\right|}=\alpha\right\},\right.\right.
$$

and the $L^{q}$-spectrum of $m$ defined by

$$
\forall q \in \mathbb{R}, \quad \tau(q)=\limsup _{n \rightarrow+\infty} \tau_{n}(q) \quad \text { with } \tau_{n}(q)=\frac{1}{n \log \ell} \log \left(\sum_{I \in \mathcal{F}_{n}} m(I)^{q}\right) .
$$

In general, we say that the multifractal formalism holds if the Hausdorff dimension dim and the Tricot dimension Dim of $E_{\alpha}$ can be computed in the following way

$$
\operatorname{dim}\left(E_{\alpha}\right)=\operatorname{Dim}\left(E_{\alpha}\right)=\tau^{*}(\alpha),
$$

where $\tau^{*}(\alpha)=\inf (\alpha q+\tau(q), q \in \mathbb{R})$ is the Legendre transform of $\tau$. In the case where $\tau$ is differentiable at a point $q_{0}$ and $\alpha=-\tau^{\prime}\left(q_{0}\right)$, we have

$$
\operatorname{dim}\left(E_{\alpha}\right)=\operatorname{Dim}\left(E_{\alpha}\right)=\alpha q_{0}+\tau\left(q_{0}\right) .
$$

There is recently been a great interest for this subject and some positive results have been written in various contexts (see for example [2-5,7,13,24,25]). In particular, if $m$ has the following quasi-Bernoulli property (see (1.2))

$$
\exists C>0, \forall(n, p) \in \mathbb{N}^{2}, \forall I \in \mathcal{F}_{n}, \forall J \in \mathcal{F}_{p}, \quad \frac{1}{C} m(I) m(J) \leqslant m(I J) \leqslant C m(I) m(J),
$$

Brown, Michon, Peyriere established in [5] that $\operatorname{dim}\left(E_{\alpha}\right)=\operatorname{Dim}\left(E_{\alpha}\right)=\tau^{*}(\alpha)$ for any $\alpha=-\tau^{\prime}(q)$. A few years later, in [13] Heurteaux showed that in this situation, $\tau$ is differentiable on $\mathbb{R}$. So, we have

$$
\forall \alpha \in\left(-\tau^{\prime}(+\infty),-\tau^{\prime}(-\infty)\right), \quad \operatorname{dim}\left(E_{\alpha}\right)=\operatorname{Dim}\left(E_{\alpha}\right)=\tau^{*}(\alpha) .
$$

Here we extend these results by introducing a new homogeneity condition which includes the above situation. More precisely, we say that $m$ has the weak quasi-Bernoulli property (see (1.3)) if for every $(n, p) \in \mathbb{N}^{2}$ and for every $I \in \mathcal{F}_{n}, J \in \mathcal{F}_{p}$ we have

$$
\frac{1}{C} m(I) \sum_{k=r_{1}}^{r_{2}} m\left(\sigma^{-k}(J)\right) \leqslant \sum_{k=p_{1}}^{p_{2}} m\left(I \cap \sigma^{-(n+k)}(J)\right) \leqslant C m(I) \sum_{k=s_{1}}^{s_{2}} m\left(\sigma^{-k}(J)\right),
$$

where $C$ is a constant which is independent of $n, p, I$ and $J$. In this context, we show the existence of a Gibbs measure $m_{q}$ at every state $q \geqslant 0$ for the measure $m$, that is

$$
\forall n, \forall I \in \mathcal{F}_{n}, \quad \frac{1}{C} m(I)^{q} \ell^{-n \tau(q)} \leqslant m_{q}(I) \leqslant C m(I)^{q} \ell^{-n \tau(q)},
$$

where $C>0$ is a constant which is independent of $n$ and $I$. Moreover, if the measure satisfies the weak quasiBernoulli property, we establish that $\tau$ is differentiable on $\mathbb{R}^{+}$. So, we have the following result:

Theorem 1. If $m$ satisfies the weak quasi-Bernoulli property then $\tau$ is differentiable on $\mathbb{R}^{+}$and the multifractal formalism holds for $m$ in the following sense:

$$
\forall \alpha \in\left(-\tau^{\prime}(+\infty),-\tau_{d}^{\prime}(0)\right), \quad \operatorname{dim}\left(E_{\alpha}\right)=\operatorname{Dim}\left(E_{\alpha}\right)=\tau^{*}(\alpha) .
$$


The introduction of this new condition is motivated by the fact that is verified by a large class of self similar measure with overlaps which generally are not quasi-Bernoulli. In particular in this work, we prove the following result:

Theorem 2. We consider $N$ similitudes $S_{i}$ defined by:

$$
\begin{aligned}
S_{i}:[0,1] & \rightarrow[0,1], \\
x & \rightarrow \pm \ell^{-\alpha_{i}} x+\beta_{i},
\end{aligned}
$$

where $\alpha_{i} \in \mathbb{N}^{*}$ and such that $[0,1]=\bigcup S_{i}([0,1])$. We assume that for each $i$, the image of the interval $[0,1]$ by $S_{i}$ is a reunion of $\ell$-adic intervals. If $\mu$ is the unique probability measure which satisfies the equation

$$
\mu=\sum_{i=1}^{N} p_{i} \mu \circ S_{i}^{-1},
$$

then $\mu$ has the weak quasi-Bernoulli property.

So, by using Theorems 1 and 2, we can deduce the differentiability of the $L^{q}$-spectrum $\tau(q)$ of $\mu$ on $\mathbb{R}^{+}$and that $\mu$ satisfies the multifractal formalism on the region defined by the $q>0$. Of course, here the iterated function system which generates $\mu$ does not verify the classical Open Set Condition (see [15]). When this condition is not satisfied, the calculation of the $L^{q}$-spectrum is often very difficult and in general we do not know if the multifractal formalism holds. Only some very particular cases have been solved by using specific properties as in the case of the golden number (see [17]). Moreover, recently new types of separation condition have been introduced to ensure the validity of the multifractal formalism in certain cases (see for example $[9,18]$ ). We can note than the similitudes considered here satisfy the finite type condition introduced by Ngai and Wang in [22] and studied by Feng [9] in the special case where the contraction ratios of the similitudes are all the same. Thus, when the contraction ratios are all the same, the multifractal analysis of $\mu$ can also be obtained, by another way, from a recent result of Feng (see [9]). Nevertheless, it seems difficult to adapt Feng's method in case where the contraction ratios are not all the same. In particular, to be able to express $\mu(\Delta)$ for an fixed interval $\Delta \in \mathcal{F}_{n}$ by using Feng's method, it would be necessary that if the contraction ratio of $S_{\sigma}=S_{i_{1}} \circ \cdots \circ S_{i_{k}}$ is approximatively $\rho^{n}$ (where $\rho=\min _{i} \ell^{-\alpha_{i}}$ ) then the length of the interval $S_{\sigma}^{-1}(\Delta)$ is independent of $\sigma$. This argument, used by Feng to establish Lemma 3.2 of [9] which is essential in his analysis, is false when the contraction ratios are not all the same. In fact, to our best knowledge, there is no general result which ensures both the differentiability of the function $\tau$ and the validity of the multifractal formalism for self similar measures generated by similitudes with overlaps if the contraction ratios are not all the same.

This type of measures can be used to estimate the Hausdorff dimension of some self affine graphs. By considering a self affine graph $\Gamma_{f}$ as an attractor of an iterated function system, we can write

$$
\Gamma_{f}=\bigcup_{i} A_{i}\left(\Gamma_{f}\right)
$$

where the maps $A_{i}$ are contractive affinities on the unit square. To obtain a lower bound of the dimension of $\Gamma_{f}$, we can introduce the probability measure $v$ such that

$$
v=\sum q_{i} v \circ A_{i}^{-1}
$$

and we try to estimate its dimension denoted by $\operatorname{dim}(\nu)$. In particular, if $\tau_{\nu}^{\prime}(1)$ exists, we have $\operatorname{dim}(\nu)=-\tau_{\nu}^{\prime}(1)$ (see $[8,13,21]$ ). One particularity of the self affine context is that we can easily link $\tau_{v}$ with $\tau_{v_{x}}$ and $\tau_{v_{y}}$ (see Proposition 4.2 and Theorem 4.1). Moreover, in some classical situations, $v_{x}$ is a self similar measure which satisfies the Open Set Condition and $v_{y}$ is of the same type as the self similar measures described above. So, in the last part of this work, we give a new approach of examples studied before by McMullen [20], Przytycki and Urbański [26,30], Kenyon and Peres [16]. 


\section{Introduction}

Dans tout ce travail on fixe un entier $\ell$ supérieur ou égal à deux et on note $\mathcal{F}_{n}$ la famille des intervalles $\ell$-adiques de la nième génération inclus dans l'intervalle $[0,1[$. En d'autres termes,

$$
\mathcal{F}_{n}=\left\{I=\left[k / \ell^{n},(k+1) / \ell^{n}\left[; 0 \leqslant k<\ell^{n}\right\} .\right.\right.
$$

De plus, si $\epsilon_{1}, \ldots, \epsilon_{n} \in\{0, \ldots, \ell-1\}^{n}$ on note $I_{\epsilon_{1} \ldots \epsilon_{n}}$ l'intervalle dyadique de nième génération :

$$
I_{\epsilon_{1} \ldots \epsilon_{n}}=\left[\sum_{i=1}^{n} \frac{\epsilon_{i}}{\ell^{i}}, \sum_{i=1}^{n} \frac{\epsilon_{i}}{\ell^{i}}+\frac{1}{2^{n}}[.\right.
$$

Par souci de simplification, si $I=I_{\epsilon_{1} \ldots \epsilon_{n}}$ et $J=I_{\epsilon_{n+1} \ldots \epsilon_{n+p}}$, on notera $I J=I_{\epsilon_{1} \ldots \epsilon_{n+p}}$. Par ailleurs pour tout $x \in$ $\left[0,1\left[\right.\right.$, on note $I_{n}(x)$ l'unique élément de $\mathcal{F}_{n}$ auquel $x$ appartient et $\left|I_{n}(x)\right|$ sa longueur.

On note $\sigma$ le shift en base $\ell$ sur l'intervalle [0,1[. Autrement dit,

$$
\begin{aligned}
\sigma:[0,1[ & \rightarrow[0,1[, \\
x & \rightarrow \ell x(\bmod 1) .
\end{aligned}
$$

Si $m$ est une mesure de probabilité sur $\mathbb{R}^{2}, m_{x}$ et $m_{y}$ désignent respectivement les mesures images de $m$ par les projections $\pi_{x}:(x, y) \rightarrow x$ et $\pi_{y}:(x, y) \rightarrow y$.

Enfin, lorsque pour deux fonctions $u$ et $v$, il existe une constante positive $C$ vérifiant $\frac{1}{C} u \leqslant v \leqslant C u$, on écrit $u \approx v$.

Etant donnée une mesure de probabilité $m$ sur [0,1[, l'analyse multifractale cherche à fournir une formule théorique explicite reliant la dimension des ensembles $E_{\alpha}$ où

$$
\forall \alpha>0, \quad E_{\alpha}=\left\{x \in \left[0,1\left[, \lim _{n \rightarrow+\infty} \frac{\log m\left(I_{n}(x)\right)}{\log \left|I_{n}(x)\right|}=\alpha\right\}\right.\right.
$$

et la fonction $\tau$ définie par

$$
\forall q \in \mathbb{R}, \quad \tau(q)=\limsup _{n \rightarrow+\infty} \tau_{n}(q) \quad \text { avec } \tau_{n}(q)=\frac{1}{n \log \ell} \log \left(\sum_{I \in \mathcal{F}_{n}} m(I)^{q}\right)
$$

(on a pris comme convention $0^{q}=0$ ). Généralement, on dit que $m$ vérifie le formalisme multifractal si on a la relation

$$
\operatorname{dim}\left(E_{\alpha}\right)=\operatorname{Dim}\left(E_{\alpha}\right)=\tau^{*}(\alpha)
$$

où $\tau^{*}(\alpha)=\inf (\alpha q+\tau(q), q \in \mathbb{R})$ désigne la transformée de Legendre de la fonction $\tau$, dim et Dim les dimensions de Hausdorff et de Tricot (on peut consulter [29] pour plus de détails).

Au cours de la dernière décennie, de nombreux travaux ont été entrepris dans ce sens (voir par exemple [2-5, $7,13,24,25])$. En particulier un des premiers travaux sur ce thème est dû à Brown, Michon et Peyrière (voir [5]). Ils ont introduit une condition d'homogénéité assurant la validité du formalisme multifractal au sens précisé par la formule (1.1). De manière plus précise, ils ont étudié les mesures $m$ quasi-Bernoulli, c'est à dire vérifiant

$$
\forall(n, p) \in \mathbb{N}^{2}, \forall I \in \mathcal{F}_{n}, \forall J \in \mathcal{F}_{p}, \quad \frac{1}{C} m(I) m(J) \leqslant m(I J) \leqslant C m(I) m(J),
$$

où $C$ est une constante positive indépendante de $n, p, I$ et $J$. Dans [19], Michon a montré que dans cette situation $m$ possède des mesures de Gibbs pour tout état. Autrement dit, pour tout $q \in \mathbb{R}$, il existe une mesure $v_{q}$ telle que

$$
\forall n, \forall I \in \mathcal{F}_{n}, \quad \frac{1}{C} m(I)^{q}|I|^{\tau(q)} \leqslant v_{q}(I) \leqslant C m(I)^{q}|I|^{\tau(q)},
$$


où $C$ est une constante positive indépendante de $n$ et $I$. Dans [5], Brown, Michon et Peyrière ont remarqué que lorsque $\tau$ est dérivable au point $q, v_{q}$ est portée par $E_{-\tau^{\prime}(q)}$. A partir de cela, ils ont alors pu établir la relation (1.1) pour les $\alpha$ pouvant s'écrire $\alpha=-\tau^{\prime}(q)$.

Plus tard, en reliant dans [13] les dérivées à gauche et à droite de la fonction $\tau$ au point 1 avec les dimensions des ensembles de pleine mesure pour $m$, Heurteaux a prouvé que dans la situation quasi-Bernoulli, $\tau$ est dérivable sur $\mathbb{R}$. Tout cela donne finalement le résultat suivant :

$$
\forall \alpha \in\left(-\tau^{\prime}(+\infty),-\tau^{\prime}(-\infty)\right), \quad \operatorname{dim}\left(E_{\alpha}\right)=\operatorname{Dim}\left(E_{\alpha}\right)=\tau^{*}(\alpha) .
$$

Ici, nous allons étendre les résultats de Brown, Michon, Peyrière en introduisant la condition d'homogénéité plus générale dite quasi-Bernoulli au sens faible. Une mesure $m$ est quasi-Bernoulli au sens faible s'il existe des entiers $r_{1}, r_{2}, p_{1}, p_{2}, s_{1}, s_{2}$ tels que pour tout couple d'entiers $(n, p)$ et pour tout intervalle $I \in \mathcal{F}_{n}, J \in \mathcal{F}_{p}$, on a

$$
\frac{1}{C} m(I) \sum_{k=r_{1}}^{r_{2}} m\left(\sigma^{-k}(J)\right) \leqslant \sum_{k=p_{1}}^{p_{2}} m\left(I \cap \sigma^{-(n+k)}(J)\right) \leqslant C m(I) \sum_{k=s_{1}}^{s_{2}} m\left(\sigma^{-k}(J)\right),
$$

où $C$ est une constante positive indépendante de $n, p, I$ et $J$. Dans cette situation, on va montrer que $m$ possède des mesures de Gibbs aux états positifs et que $\tau$ est dérivable sur $\mathbb{R}^{+}$. Par suite, on déduira qu'une mesure $m$ quasi-Bernoulli au sens faible vérifie le formalisme multifractal au sens suivant :

$$
\forall q>0, \quad \operatorname{dim}\left(E_{-\tau^{\prime}(q)}\right)=\operatorname{Dim}\left(E_{-\tau^{\prime}(q)}\right)=\tau^{*}\left(-\tau^{\prime}(q)\right)=-q \tau^{\prime}(q)+\tau(q) .
$$

En particulier, la dérivabilité de $\tau$ au point 1 nous assure que la mesure $m$ est unidimensionnelle de dimension $-\tau^{\prime}(1)$. Cela peut par exemple être vu comme une conséquence des travaux de Fan, Heurteaux, Ngai (voir [8,13, 21]) qui relient la dimension d'une mesure avec la dérivée de sa fonction $\tau$ au point 1.

Plus précisément, pour une mesure de probabilité $m$ sur $[0,1]^{d}$, on définit respectivement la dimension inférieure et supérieure de $m$ par:

$$
\left\{\begin{array}{l}
\operatorname{dim}_{*}(m):=\inf \{\operatorname{dim}(E), m(E)>0\}, \\
\operatorname{dim}^{*}(m):=\inf \left\{\operatorname{dim}(E), m\left([0,1]^{d} \backslash E\right)=0\right\} .
\end{array}\right.
$$

On peut définir le même type de quantités pour la dimension de Tricot Dim. $\operatorname{Lorsque}_{\operatorname{dim}}(m)=\operatorname{dim}^{*}(m)=$ $\operatorname{Dim}_{*}(m)=\operatorname{Dim}^{*}(m)$, on dit que $m$ est unidimensionnelle et on appelle ce nombre la dimension de $m$ que l'on $\operatorname{note} \operatorname{dim}(m)$ (pour plus de détails, on peut consulter $[7,8,13,27]$ ).

L'introduction de cette nouvelle condition est motivée par le fait qu'elle est vérifiée par une large classe de mesures autosimilaires qui en général ne satisfont pas la propriété quasi-Bernoulli. En particulier, on considère dans ce papier $N$ similitudes $S_{i}:[0,1] \rightarrow[0,1]$ dont les images sont des réunions d'intervalles $\ell$-adiques et de rapport de contraction respectif $\pm \ell^{-\alpha_{i}}$ où $\alpha_{i} \in \mathbb{N}^{*}$. De plus, on suppose que $[0,1]=\bigcup S_{i}([0,1])$. On montre alors que l'unique mesure de probabilité $\mu$ vérifiant

$$
\mu=\sum_{i=1}^{N} p_{i} \mu \circ S_{i}^{-1}
$$

est quasi-Bernoulli au sens faible. De cela, on déduit notamment la dérivabilité de la fonction $\tau$ sur $\mathbb{R}^{+}$et le fait que la mesure $\mu$ satisfait le formalisme multifractal au sens précisé par la relation (1.4).

Evidemment, les images des similitudes considérées ici ne vérifient pas la condition de l'ensemble ouvert (voir [15]). Plus précisément, celles-ci peuvent avoir des intersections d'intérieur non vide, voir même pour certaines d'entre elles être incluses les unes dans les autres. On parle alors de phénomène d'overlapping. En général, dans cette situation il est très difficile d'effectuer le calcul de la fonction $\tau$ et on ne sait pas si le formalisme multifractal fonctionne. Seuls quelques cas très particuliers ont pu être traités explicitement en utilisant par exemple des propriétés algébriques (voir entre autres le cas du nombre d'or dans [17]). Par ailleurs, les travaux de Lau et 
Ngai [18] et de Feng [9] ont permis de dégager des conditions de séparabilité plus faibles assurant la validité du formalisme multifractal dans certains cas. On note que les systèmes de fonctions itérées que l'on étudie satisfont la condition de type fini introduite par Ngai et Wang dans [22] et reprise par Feng [9] dans le cas particulier où les rapports de contraction des similitudes sont tous égaux. Ainsi, lorsque tous les rapports de contraction sont égaux, la dérivabilité de la fonction $\tau$ sur $\mathbb{R}^{+}$et l'analyse multifractale de $\mu$ pour les $q>0$ peuvent aussi être obtenues, avec une approche différente, en utilisant un récent résultat de Feng (voir [9]). En revanche, il semble difficile d'adapter le raisonnement de Feng au cas où les rapports de contractions diffèrent les uns des autres. En effet, pour exprimer la masse d'un intervalle $\Delta$ de nième génération selon la méthode de Feng, il faudrait utiliser le fait que si $S_{\sigma}=S_{i_{1}} \circ \cdots \circ S_{i_{k}}$ a un rapport de contraction de l'ordre de $\rho^{n}$ alors la longueur de l'intervalle $S_{\sigma}^{-1}(\Delta)$ est indépendante de $\sigma\left(\rho:=\min _{1} \ell^{-\alpha_{i}}\right.$ étant le plus petit rapport de contraction des similitudes considérées). Cet argument, utilisé par Feng pour établir la relation (3.2) qui conduit au Lemme 3.2 de [9], essentiel dans sa démarche, n'est plus vérifié lorsque les rapports de contraction ne sont pas tous égaux (voir remarque à propos du Corollaire 3.1). En fait, à notre connaissance, il ne semble pas qu'il existe de résultat général assurant à la fois la dérivabilité de la fonction $\tau$ et la validité du formalisme multifractal pour des mesures autosimilaires définies par des similitudes possédant des overlaps et dont les rapports de contraction ne sont pas forcément tous égaux.

Ce type de mesures apparaît par exemple lorsqu'on cherche à estimer la dimension de certains graphes autoaffines (voir $[16,20,26,30])$. En voyant un graphe autoaffine $\Gamma_{f}$, comme attracteur d'un système de fonctions itérées, on a

$$
\Gamma_{f}=\bigcup_{i} A_{i}\left(\Gamma_{f}\right)
$$

où les applications $A_{i}$ sont des affinités du carré unité dans lui même. Pour minorer $\operatorname{dim}\left(\Gamma_{f}\right)$, on peut introduire la mesure autoaffine portée par $\Gamma_{f}$ vérifiant l'équation suivante

$$
v=\sum q_{i} v \circ A_{i}^{-1}
$$

dont on cherche à estimer la dimension.

Une des particularités du contexte autoaffine est que l'on peut souvent relier la dimension de $v$ avec les dimensions de ses mesures projetées $v_{x}$ et $v_{y}$. La mesure $v_{x}$ étant une mesure autosimilaire sans overlapping, toute la difficulté consiste en fait à étudier la mesure $v_{y}$. On constatera notamment que sur certains exemples classiques, la mesure $v_{y}$ est du même type que les mesures autosimilaires avec overlapping évoquées précédemment. On insistera notamment sur les cas où $v_{x}$ et $v_{y}$ sont absolument continues par rapport à la mesure de Lebesgue. Cette condition fournit en effet des situations où la dimension de Hausdorff et la dimension de Tricot du graphe coïncident (voir [30]).

Le papier est organisé de la manière suivante :

Au cours d'une première partie, on énonce et on démontre le résultat principal :

Théorème 1.1. Si $m$ est quasi-Bernoulli au sens faible alors $\tau$ est dérivable sur $\mathbb{R}^{+}$et $m$ vérifie le formalisme multifractal au sens suivant :

$$
\forall \alpha \in\left(-\tau^{\prime}(+\infty),-\tau_{d}^{\prime}(0)\right), \quad \operatorname{dim}\left(E_{\alpha}\right)=\operatorname{Dim}\left(E_{\alpha}\right)=\tau^{*}(\alpha) .
$$

Ensuite, la deuxième partie est consacrée à la mise en évidence de mesures autosimilaires avec overlapping possédant la propriété quasi-Bernoulli au sens faible. On montre notamment le résultat suivant : 
Théorème 1.2. On considère $N$ similitudes $S_{i}:[0,1] \rightarrow[0,1]$ de rapport de contraction respectif $\pm \ell^{-\alpha_{i}}$ où $\alpha_{i} \in \mathbb{N}^{*}$ et dont les images sont des réunions d'intervalles $\ell$-adiques. On suppose de plus que $[0,1]=\bigcup_{i} S_{i}([0,1])$. Alors l'unique mesure de probabilité $\mu$ vérifiant:

$$
\mu=\sum_{i=1}^{N} p_{i} \mu \circ S_{i}^{-1}
$$

est quasi-Bernoulli au sens faible.

Les Théorèmes 1.1 et 1.2 fournissent alors des informations sur l'analyse multifractale de la mesure $\mu$.

Enfin, lors de la troisième partie on évoque le lien entre les mesures étudiées dans la deuxième partie et l'étude de certains graphes autoaffines. On propose une nouvelle approche d'exemples déjà décrits par McMullen [20], Przytycki et Urbański [26,30], Kenyon et Peres [16].

\section{Analyse multifractale des mesures quasi-Bernoulli au sens faible}

On reprend les notations déjà introduites. On rappelle qu'une mesure $m$ sur [0,1[ est quasi-Bernoulli au sens faible s'il existe une constante $C>0$ et des entiers positifs $r_{1}, r_{2}, p_{1}, p_{2}, s_{1}, s_{2}$ tels que pour tout couple d'entier $(n, p)$ et pour tout intervalle $I \in \mathcal{F}_{n}, J \in \mathcal{F}_{p}$, on a :

$$
\frac{1}{C} m(I) \sum_{k=r_{1}}^{r_{2}} m\left(\sigma^{-k}(J)\right) \leqslant \sum_{k=p_{1}}^{p_{2}} m\left(I \cap \sigma^{-(n+k)}(J)\right) \leqslant C m(I) \sum_{k=s_{1}}^{s_{2}} m\left(\sigma^{-k}(J)\right) .
$$

Certaines mesures étudiées dans [11] vérifient une condition qui peut être vue comme un cas particulier de cette généralisation. De plus, en prenant $r_{1}=r_{2}=p_{1}=p_{2}=s_{1}=s_{2}=0$, on retrouve exactement la condition quasi-Bernoulli (1.2) car si $I \in \mathcal{F}_{n}, I \cap \sigma^{-n}(J)$ n'est autre que $I J$.

Dans cette situation, on va montrer que $m$ possède des mesures de Gibbs aux états positifs et que $\tau$ est dérivable sur $] 0,+\infty[$. Il en résultera que :

$$
\forall \alpha \in\left(-\tau^{\prime}(+\infty),-\tau_{d}^{\prime}(0)\right), \quad \operatorname{dim}\left(E_{\alpha}\right)=\operatorname{Dim}\left(E_{\alpha}\right)=\tau^{*}(\alpha) .
$$

Sauf mention contraire, dans toute la suite de cette partie, $I$ désigne un intervalle $\ell$-adique de génération $n, J$ un intervalle $\ell$-adique quelconque et on note indifféremment $C$ toute constante dépendant des constantes $r_{1}, r_{2}$, $p_{1}, p_{2}, s_{1}, s_{2}, \ell$ introduites précédemment et de $q$ que l'on aura fixé, mais indépendante des générations $n$ et $p$.

Théorème 2.1. Soit $m$ une mesure de probabilité sur $[0,1$ [ quasi-Bernoulli au sens faible (voir (1.3)). Alors $m$ admet des mesures de Gibbs aux états positifs, c'est à dire pour tout $q \geqslant 0$, il existe une mesure de probabilité $v$ vérifiant :

$$
\exists C>0, \forall n, \forall I \in \mathcal{F}_{n}, \quad \frac{1}{C} m(I)^{q}|I|^{\tau(q)} \leqslant \nu(I) \leqslant C m(I)^{q}|I|^{\tau(q)} .
$$

Remarque. Contrairement au cas d'une mesure quasi-Bernoulli qui admet des mesures de Gibbs pour tout état réel, ici on a seulement l'existence de telles mesures aux états positifs. En particulier, le Lemme 2.1 énoncé dans la preuve du théorème 2.1 est faux si $q<0$.

\section{Preuve du Théorème 2.1.}

Etape 1. On fixe $q \geqslant 0$. On montre tout d'abord que la limite de la suite $\tau_{n}(q)$ existe et est finie. En d'autres termes, 


$$
\tau(q)<\infty \quad \text { et } \quad \tau(q)=\lim _{n \rightarrow \infty} \tau_{n}(q) .
$$

De plus, l'encadrement suivant est vérifié

$$
\frac{1}{C} \ell^{n \tau(q)} \leqslant \ell^{n \tau_{n}(q)} \leqslant C \ell^{n \tau(q)}
$$

où $C$ est une constante indépendante de $n$.

Ceci repose essentiellement sur les propriétés de convergence des suites sur(ou sous)-multiplicatives et sur le lemme classique suivant :

Lemme 2.1. Pour tout réel $q \geqslant 0$ et pour tout entier $k \geqslant 1$, il existe une constante $C>0$ telle que si $a_{1}, \ldots, a_{k} \in$ $\mathbb{R}^{+}$alors on $a$ :

$$
\frac{1}{C} \sum_{i=1}^{k} a_{i}^{q} \leqslant\left(\sum_{i=1}^{k} a_{i}\right)^{q} \leqslant C \sum_{i=1}^{k} a_{i}^{q} .
$$

(On peut prendre $C=\max \left(k^{q-1}, 1\right)$.)

Du Lemme 2.1, on déduit le Lemme 2.2 qui donne un encadrement qui sera utile tout au long de la preuve.

Lemme 2.2. Pour tout réel $q \geqslant 0$ et pour tout entier $k \geqslant 1$, il existe une constante $C>0$ telle que pour tout entier $n \geqslant 0$, on $a$ :

$$
\frac{1}{C} \sum_{I \in \mathcal{F}_{n}} m(I)^{q} \leqslant \sum_{I \in \mathcal{F}_{n+k}} m(I)^{q} \leqslant C \sum_{I \in \mathcal{F}_{n}} m(I)^{q} .
$$

Preuve du Lemme 2.2. Grâce au Lemme 2.1, on a :

$$
\sum_{I \in \mathcal{F}_{n+k}} m(I)^{q}=\sum_{I \in \mathcal{F}_{n}} \sum_{K \in \mathcal{F}_{k}} m(I K)^{q} \leqslant C \sum_{I \in \mathcal{F}_{n}}\left(\sum_{K \in \mathcal{F}_{k}} m(I K)\right)^{q}=C \sum_{I \in \mathcal{F}_{n}} m(I)^{q} .
$$

De manière similaire, on obtient l'autre inégalité, ce qui prouve le Lemme 2.2.

Montrons maintenant (2.2) et (2.3). D'après le Lemme 2.2, on a

$$
u_{n+p}:=\sum_{K \in \mathcal{F}_{n+p}} m(K)^{q} \leqslant C \sum_{k=p_{1}}^{p_{2}} \sum_{K \in \mathcal{F}_{n+p+k}} m(K)^{q}=C \sum_{k=p_{1}}^{p_{2}} \sum_{I \in \mathcal{F}_{n}} \sum_{J \in \mathcal{F}_{p}} \sum_{K \in \mathcal{F}_{k}} m(I K J)^{q},
$$

où $C$ est une constante indépendante de $n$ et de $p$. Comme $m$ vérifie (1.3), on obtient grâce au Lemme 2.1 :

$$
\begin{aligned}
u_{n+p} & \leqslant C \sum_{I \in \mathcal{F}_{n}} \sum_{J \in \mathcal{F}_{p}}\left(\sum_{k=p_{1}}^{p_{2}} \sum_{K \in \mathcal{F}_{k}} m(I K J)\right)^{q} \leqslant C \sum_{I \in \mathcal{F}_{n}} m(I)^{q} \sum_{J \in \mathcal{F}_{p}}\left(\sum_{k=s_{1}}^{s_{2}} m\left(\sigma^{-k}(J)\right)\right)^{q} \\
& \leqslant C \sum_{I \in \mathcal{F}_{n}} \sum_{J \in \mathcal{F}_{p}} m(I)^{q} \sum_{k=s_{1}}^{s_{2}} m\left(\sigma^{-k}(J)\right)^{q} .
\end{aligned}
$$

En utilisant à nouveau le Lemme 2.2, on trouve

$$
u_{n+p} \leqslant C \sum_{I \in \mathcal{F}_{n}} m(I)^{q} \sum_{k=s_{1}}^{s_{2}} \sum_{J \in \mathcal{F}_{p+k}} m(J)^{q} \leqslant C \sum_{I \in \mathcal{F}_{n}} m(I)^{q} \sum_{J \in \mathcal{F}_{p}} m(J)^{q}=C u_{n} u_{p} .
$$


Ainsi, la suite $C u_{n}$ est sous-multiplicative. Quitte à modifier la constante $C$, on montrerait de même que la suite $\frac{1}{C} u_{n}$ est sur-multiplicative.

Les relations (2.2) et (2.3) sont alors classiques, la suite $C^{1 / n} \ell^{\tau_{n}(q)}$ convergeant vers sa borne inférieure et la suite $\left(\frac{1}{C}\right)^{1 / n} \ell^{\tau_{n}(q)}$ convergeant vers sa borne supérieure.

Etape 2. Construction de la mesure de Gibbs.

Pour simplifier les écritures, on suppose que pour tout $i \in\{0, \ldots, \ell-1\}, \mu\left(I_{i}\right)>0$, ce qui entraîne d'après (1.3), $\mu(I)>0$ pour tout $I$ intervalle $\ell$-adique. (Si ce n'était pas le cas, il suffirait de changer d'alphabet.)

On considère $v_{n}$ une mesure de probabilité, à densité par rapport à la mesure de Lebesgue sur [0,1], telle que

$$
\forall I \in \mathcal{F}_{n}, \quad v_{n}(I)=\lambda m(I)^{q} .
$$

Comme $\sum_{I \in \mathcal{F}_{n}} v_{n}(I)=1$, on a $\lambda=\ell^{-n \tau_{n}(q)}=|I|^{\tau_{n}(q)}$ d'où finalement :

$$
\forall I \in \mathcal{F}_{n}, \quad v_{n}(I)=|I|^{\tau_{n}(q)} m(I)^{q} .
$$

Pour $I \in \mathcal{F}_{n}$ et $p \geqslant p_{2}$, on a ainsi :

$$
v_{n+p}(I)=\frac{1}{p_{2}-p_{1}+1} \sum_{k=p_{1}}^{p_{2}} \sum_{K \in \mathcal{F}_{k}} \sum_{J \in \mathcal{F}_{p-k}} v_{n+p}(I K J) \leqslant C \sum_{k=p_{1}}^{p_{2}} \sum_{K \in \mathcal{F}_{k}} \sum_{J \in \mathcal{F}_{p-k}} m(I K J)^{q}|I K J|^{\tau_{n+p}(q)} .
$$

Or d'après (2.3) on a

$$
|I K J|^{\tau_{n+p}(q)}=\ell^{-(n+p) \tau_{n+p}(q)} \leqslant C \ell^{-n \tau_{n}(q)} \ell^{-p \tau_{p}(q)},
$$

ce qui donne

$$
v_{n+p}(I) \leqslant C \ell^{-n \tau_{n}(q)} \ell^{-p \tau_{p}(q)} \sum_{k=p_{1}}^{p_{2}} \sum_{K \in \mathcal{F}_{k}} \sum_{J \in \mathcal{F}_{p-k}} m(I K J)^{q} .
$$

Grâce au Lemme 2.2, on a donc

$$
v_{n+p}(I) \leqslant C \ell^{-n \tau_{n}(q)} \ell^{-p \tau_{p}(q)} \sum_{J \in \mathcal{F}_{p}} \sum_{k=p_{1}}^{p_{2}} \sum_{K \in \mathcal{F}_{k}} m(I K J)^{q} .
$$

De plus, le Lemme 2.1 et le fait que $m$ vérifie (1.3) impliquent :

$$
\sum_{k=p_{1}}^{p_{2}} \sum_{K \in \mathcal{F}_{k}} m(I K J)^{q} \leqslant C\left(\sum_{k=p_{1}}^{p_{2}} \sum_{K \in \mathcal{F}_{k}} m(I K J)\right)^{q} \leqslant C m(I)^{q} \sum_{k=s_{1}}^{s_{2}} m\left(\sigma^{-k}(J)\right)^{q} .
$$

En injectant (2.7) dans (2.6), on obtient

$$
v_{n+p}(I) \leqslant C m(I)^{q} \ell^{-n \tau_{n}(q)} \ell^{-p \tau_{p}(q)} \sum_{J \in \mathcal{F}_{p}} \sum_{k=s_{1}}^{s_{2}} m\left(\sigma^{-k}(J)\right)^{q} \leqslant C m(I)^{q} \ell^{-n \tau_{n}(q)} \ell^{-p \tau_{p}(q)} \sum_{k=s_{1}}^{s_{2}} \sum_{J \in \mathcal{F}_{p+k}} m(J)^{q} .
$$

Par suite, d'après le Lemme 2.2,

$$
v_{n+p}(I) \leqslant C m(I)^{q}|I|^{\tau_{n}(q)} \sum_{J \in \mathcal{F}_{p}} m(J)^{q}|J|^{\tau_{p}(q)}=C m(I)^{q}|I|^{\tau_{n}(q)} ;
$$

finalement, on déduit de (2.3) qu'il

$$
\exists C>0, \forall n \in \mathbb{N}^{*}, \forall I \in \mathcal{F}_{n}, \forall p \in \mathbb{N}^{*}, \quad v_{n+p}(I) \leqslant C m(I)^{q}|I|^{\tau(q)} .
$$


On montrerait en utilisant les mêmes techniques une minoration du même type. Ainsi en considérant une limite faible $v$ de la suite $v_{n}$, on obtient une mesure de Gibbs pour la mesure $m$ à l'état $q$, ce qui prouve le Théorème 2.1 .

Théorème 2.2. Soit $m$ une mesure de probabilité sur [0, 1[ quasi-Bernoulli au sens faible. Alors $\tau$ est dérivable au point 1.

Preuve du Théorème 2.2. Esquissons tout d'abord le fil conducteur de la preuve. Il s'agit d'adapter à notre situation un raisonnement utilisé dans [13] pour les mesures quasi-Bernoulli. On rappelle que deux mesures $m_{1}$ et $m_{2}$ sont dites fortement équivalentes s'il existe une constante $C>0$ telle que $\frac{1}{C} m_{1} \leqslant m_{2} \leqslant C m_{1}$. On va en fait construire une mesure $\tilde{m}$ fortement équivalente à $\mu:=\frac{1}{t_{2}-t_{1}+1} \sum_{k=t_{1}}^{t_{2}} m \circ \sigma^{-k}$ où $t_{1}$ et $t_{2}$ sont des entiers positifs ne dépendant que de $r_{1}, r_{2}, p_{1}, p_{2}, s_{1}, s_{2}$ qui sera $\sigma$ invariante et ergodique. De plus, elle vérifiera $\operatorname{dim}_{*}(\tilde{m})=$ $-\left(\tau_{\tilde{m}}\right)_{d}^{\prime}(1)$ et $\operatorname{Dim}^{*}(\tilde{m})=-\left(\tau_{\tilde{m}}\right)_{g}^{\prime}(1)$ où $\tau_{\widetilde{m}}$ est la fonction $\tau$ associée à $\widetilde{m}$. L'utilisation du théorème de ShannonMcMillan (on peut consulter [1]) entraînera alors la dérivabilité de $\tau_{\tilde{m}}$ au point 1 . Enfin, on constatera que sur $\mathbb{R}^{+}$, $\tau_{\widetilde{m}}=\tau_{m}$.

Soit $\widetilde{m}$ une valeur d'adhérence de la suite de mesures :

$$
m_{n}=\frac{1}{n} \sum_{k=0}^{n-1} m \circ \sigma^{-k}
$$

Soit $I \in \mathcal{F}_{n}$. Remarquons que l'on peut modifier la deuxième inégalité de (1.3) de la manière suivante :

$$
\sum_{k=p_{1}+1}^{p_{2}+1} m\left(I \cap \sigma^{-(n+k)}(J)\right) \leqslant C m(I) \sum_{k=s_{1}+1}^{s_{2}+1} m\left(\sigma^{-k}(J)\right) .
$$

En effet,

$$
\begin{aligned}
\sum_{k=p_{1}+1}^{p_{2}+1} m\left(I \cap \sigma^{-(n+k)}(J)\right) & =\sum_{k=p_{1}+1}^{p_{2}+1} \sum_{K \in \mathcal{F}_{k-1}} \sum_{L \in \mathcal{F}_{1}} m(I K L J)=\sum_{L \in \mathcal{F}_{1}} \sum_{k=p_{1}}^{p_{2}} \sum_{K \in \mathcal{F}_{k}} m(I K L J) \\
& \leqslant C m(I) \sum_{L \in \mathcal{F}_{1}} \sum_{k=s_{1}}^{s_{2}} m\left(\sigma^{-k}(L J)\right)=C m(I) \sum_{k=s_{1}+1}^{s_{2}+1} m\left(\sigma^{-k}(J)\right) .
\end{aligned}
$$

On peut procéder de même pour la première inégalité de (1.3). En itérant cette remarque, on peut alors trouver facilement des entiers naturels $t_{1}, t_{2}, p_{1}^{\prime}, p_{2}^{\prime}, p_{1}^{\prime \prime}, p_{2}^{\prime \prime}$ ne dépendant pas de $I$ et $J$ tels que :

$$
\left\{\begin{array}{l}
\frac{1}{C} m(I) \sum_{k=t_{1}}^{t_{2}} m\left(\sigma^{-k}(J)\right) \leqslant \sum_{k=p_{1}^{\prime \prime}}^{p_{2}^{\prime \prime}} m\left(I \cap \sigma^{-(n+k)}(J)\right), \\
\sum_{k=p_{1}^{\prime}}^{p_{2}^{\prime}} m\left(I \cap \sigma^{-(n+k)}(J)\right) \leqslant C m(I) \sum_{k=t_{1}}^{t_{2}} m\left(\sigma^{-k}(J)\right) .
\end{array}\right.
$$

Pour cela, on commence par transformer (1.3), à l'aide du procédé que l'on vient de décrire, pour modifier $r_{1}$ et $s_{1}$ en $t_{1}=\max \left(r_{1}, s_{1}\right)$. Ensuite si les "nouvelles" constantes $r_{2}$ et $s_{2}$ vérifient $s_{2} \leqslant r_{2}$, on conclut facilement en prenant $t_{2}=r_{2}$. En revanche si $r_{2}<s_{2}$, on peut par le procédé précédent obtenir successivement des majorations de

$$
m(I) \sum_{k=t_{1}}^{r_{2}} m\left(\sigma^{-k}(J)\right), \quad m(I) \sum_{k=t_{1}+1}^{r_{2}+1} m\left(\sigma^{-k}(J)\right), \ldots, m(I) \sum_{k=t_{1}+\left(s_{2}-r_{2}\right)}^{s_{2}} m\left(\sigma^{-k}(J)\right) .
$$


En sommant ces inégalités, on obtient ainsi une majoration de $m(I) \sum_{k=t_{1}}^{s_{2}} m\left(\sigma^{-k}(J)\right)$ et on peut donc prendre $t_{2}=s_{2}$.

Pour $p>p_{1}^{\prime}$ et pour tout intervalle $\ell$-adique $J$, on a donc d'après (2.9),

$$
\begin{aligned}
\sum_{k=0}^{p_{2}^{\prime}-p_{1}^{\prime}} m \circ \sigma^{-(p+k)}(J) & =\sum_{k=0}^{p_{2}^{\prime}-p_{1}^{\prime}} \sum_{I \in \mathcal{F}_{p-p_{1}^{\prime}}} \sum_{K \in \mathcal{F}_{p_{1}^{\prime}+k}} m(I K J)=\sum_{I \in \mathcal{F}_{p-p_{1}^{\prime}}} \sum_{k=p_{1}^{\prime}}^{p_{2}^{\prime}} \sum_{K \in \mathcal{F}_{k}} m(I K J) \\
& \leqslant C \sum_{I \in \mathcal{F}_{p-p_{1}^{\prime}}} m(I) \sum_{k=t_{1}}^{t_{2}} m\left(\sigma^{-k}(J)\right)=C \sum_{k=t_{1}}^{t_{2}} m\left(\sigma^{-k}(J)\right) .
\end{aligned}
$$

Par suite, on en déduit facilement que

$$
\forall J, \quad \tilde{m}(J) \leqslant C \sum_{k=t_{1}}^{t_{2}} m \circ \sigma^{-k}(J) .
$$

De même, on prouve que

$$
\forall J, \quad \tilde{m}(J) \geqslant \frac{1}{C} \sum_{k=t_{1}}^{t_{2}} m \circ \sigma^{-k}(J),
$$

ce qui montre que $\tilde{m}$ est fortement équivalente à $\mu=\frac{1}{t_{2}-t_{1}+1} \sum_{k=t_{1}}^{t_{2}} m \circ \sigma^{-k}$.

Par construction $\tilde{m}$ est $\sigma$ invariante. Ensuite $\tilde{m}$ est ergodique. En effet, pour $I \in \mathcal{F}_{n}$, en posant $\delta=$ $1 /\left(t_{2}-t_{1}+1\right)$, on a

$$
\begin{aligned}
\sum_{k=p_{1}^{\prime \prime}}^{p_{2}^{\prime \prime}} \mu\left(I \cap \sigma^{-(n+k)}(J)\right) & =\sum_{k=p_{1}^{\prime \prime}}^{p_{2}^{\prime \prime}} \sum_{K \in \mathcal{F}_{k}} \mu(I K J)=\delta \sum_{k=p_{1}^{\prime \prime}}^{p_{2}^{\prime \prime}} \sum_{K \in \mathcal{F}_{k}} \sum_{t=t_{1}}^{t_{2}} \sum_{T \in \mathcal{F}_{t}} m(T I K J) \\
& =\delta \sum_{t=t_{1}}^{t_{2}} \sum_{T \in \mathcal{F}_{t}} \sum_{k=p_{1}^{\prime \prime}}^{p_{2}^{\prime \prime}} \sum_{K \in \mathcal{F}_{k}} m(T I K J) \geqslant \frac{1}{C} \sum_{t=t_{1}}^{t_{2}} \sum_{T \in \mathcal{F}_{t}} m(T I) \mu(J)
\end{aligned}
$$

ou encore

$$
\sum_{k=p_{1}^{\prime \prime}}^{p_{2}^{\prime \prime}} \mu\left(I \cap \sigma^{-(n+k)}(J)\right) \geqslant \frac{1}{\delta C} \mu(I) \mu(J) .
$$

Comme $\tilde{m}$ est fortement équivalente à $\mu$, on obtient que

$$
\sum_{k=p_{1}^{\prime \prime}}^{p_{2}^{\prime \prime}} \tilde{m}\left(I \cap \sigma^{-(n+k)}(J)\right) \geqslant \frac{1}{C} \tilde{m}(I) \tilde{m}(J) .
$$

Cette inégalité s'étend par un argument de classe monotone au cas où $J$ est un borélien quelconque. Par conséquent, si $B=\sigma^{-1}(B)$, on a

$$
\forall I, \quad \tilde{m}(I \cap B) \geqslant \frac{1}{C} \tilde{m}(I) \tilde{m}(B) .
$$

De même, on passe de $I$ intervalle $\ell$-adique à $I$ borélien. En prenant $I=[0,1] \backslash B$, on en déduit que $\widetilde{m}(B)=0$ ou $\widetilde{m}(B)=1$, ce qui prouve que $\tilde{m}$ est ergodique. 
Ainsi $\tilde{m}$ est $\sigma$ invariante et ergodique. D'après le théorème de Shannon-McMillan, la limite de $\left(\log \left(\widetilde{m}\left(I_{n}(X)\right)\right) /\right.$ $\log \left|I_{n}(X)\right|$ existe donc $\tilde{m}$ presque sûrement et vaut une constante $h$. Par suite, on a classiquement (voir $[7,8,13,27])$ :

$$
h=\operatorname{dim}_{*}(\tilde{m})=\operatorname{dim} *(\tilde{m})=\operatorname{Dim}_{*}(\tilde{m})=\operatorname{Dim}^{*}(\tilde{m}) .
$$

Pour pouvoir conclure à la dérivabilité de $\tau_{\widetilde{m}}$ en 1 , il reste à établir que

$$
\operatorname{dim}_{*}(\tilde{m})=-\left(\tau_{\tilde{m}}\right)_{d}^{\prime}(1) \quad \text { et } \quad \operatorname{Dim}^{*}(\tilde{m})=-\left(\tau_{\tilde{m}}\right)_{g}^{\prime}(1) .
$$

En raisonnant de la même manière que dans la preuve de l'ergodicité, on obtient que

$$
\sum_{k=p_{1}^{\prime}}^{p_{2}^{\prime}} \tilde{m}\left(I \cap \sigma^{-(n+k)}(J)\right) \leqslant C \tilde{m}(I) \tilde{m}(J) .
$$

De cela, on déduit, en utilisant la même idée que dans la première étape de la preuve du Théorème 2.1, que la suite $\left(\tau_{\tilde{m}}\right)_{n}(q)$ converge pour tout $q \geqslant 0$. De plus, on a

$$
\exists C>0, \forall n \in \mathbb{N}^{*}, \quad \sum_{I \in \mathcal{F}_{n}} \tilde{m}(I)^{q} \geqslant C^{-q} \ell^{n \tau \widetilde{m}(q)} .
$$

D'après Heurteaux (voir [13]), cela est suffisant pour donner (2.12).

Pour terminer la preuve du Théorème 2.2 , montrons que sur $\mathbb{R}^{+}, \tau_{m}=\tau_{\widetilde{m}}$. On constate tout d'abord que grâce aux Lemmes 2.1 et 2.2 :

$$
\forall k, \forall q \geqslant 0, \quad \tau_{m}(q)=\tau_{m \circ \sigma^{-k}}(q) .
$$

De plus, pour tout $q \geqslant 0$, on a

$$
\tau_{\mu}(q)=\max _{t_{1} \leqslant k \leqslant t_{2}} \tau_{m \circ \sigma^{-k}}(q),
$$

(voir par exemple [4]). On obtient donc $\tau_{m}=\tau_{\mu}$ sur $\mathbb{R}^{+}$. Enfin, on a évidemment $\tau_{\widetilde{m}}=\tau_{\mu}$ car ces deux mesures sont fortement équivalentes.

Théorème 2.3. Soit $m$ une mesure de probabilité sur [0, 1[ quasi-Bernoulli au sens faible. Alors $\tau$ est dérivable sur $] 0,+\infty[$.

Remarque. Dans [11], Feng et Lau obtiennent un énoncé similaire dans un contexte différent.

Preuve du Théorème 2.3. Soit $q>0$. Enonçons tout d'abord le lemme suivant :

Lemme 2.3. Si v est la mesure de Gibbs associée à $m$ à l'état $q$ construite précédemment alors v vérifie la propriété (1.3) avec les mêmes paramètres $r_{1}, r_{2}, p_{1}, p_{2}, s_{1}, s_{2}$.

Admettons provisoirement ce lemme et montrons qu'il permet de conclure. On reprend ici une idée utilisée dans [13]. Le fait que $v$ soit une mesure de Gibbs en $q$ implique facilement que

$$
\forall t>0, \quad \tau_{v}(t)=\tau_{m}(t q)-t \tau_{m}(q),
$$

ce qui donne en posant $u=q t$,

$$
\forall u>0, \quad \tau_{m}(u)=\tau_{\nu}\left(\frac{u}{q}\right)+\frac{u}{q} \tau_{m}(q) .
$$


Le Théorème 2.2 et le Lemme 2.3 permettent de dire que $\tau_{v}^{\prime}(1)$ existe. Par suite, $\tau_{m}$ est dérivable en $q$ et on a :

$$
\tau_{m}^{\prime}(q)=\frac{1}{q}\left(\tau_{v}^{\prime}(1)+\tau_{m}(q)\right) .
$$

Reste à établir le Lemme 2.3.

Preuve du Lemme 2.3. Montrons par exemple que :

$$
\sum_{k=p_{1}}^{p_{2}} v\left(I \cap \sigma^{-(n+k)}(J)\right) \leqslant C v(I) \sum_{k=s_{1}}^{s_{2}} v\left(\sigma^{-k}(J)\right) .
$$

(L'inégalité inverse se montre en utilisant le même argument.)

D'après (2.1), on a

$$
\sum_{k=p_{1}}^{p_{2}} v\left(I \cap \sigma^{-(n+k)}(J)\right)=\sum_{k=p_{1}}^{p_{2}} \sum_{K \in \mathcal{F}_{k}} v(I K J) \leqslant C \sum_{k=p_{1}}^{p_{2}} \sum_{K \in \mathcal{F}_{k}}|I K J|^{\tau(q)} m(I K J)^{q},
$$

comme $m$ vérifie (1.3), on obtient grâce au Lemme 2.1,

$$
\sum_{k=p_{1}}^{p_{2}} v\left(I \cap \sigma^{-(n+k)}(J)\right) \leqslant C|I|^{\tau(q)}|J|^{\tau(q)} m(I)^{q} \sum_{k=s_{1}}^{s_{2}} m\left(\sigma^{-k}(J)\right)^{q} \leqslant C v(I) \sum_{k=s_{1}}^{s_{2}} \sum_{K \in \mathcal{F}_{k}}|K J|^{\tau(q)} m(K J)^{q},
$$

ce qui donne finalement

$$
\sum_{k=p_{1}}^{p_{2}} v\left(I \cap \sigma^{-(n+k)}(J)\right) \leqslant C v(I) \sum_{k=s_{1}}^{s_{2}} v\left(\sigma^{-k}(J)\right) .
$$

Ceci prouve le Lemme 2.3 et termine la preuve du Théorème 2.3.

On peut maintenant énoncer le théorème suivant qui découle immédiatement des Théorèmes 2.1 et 2.3 et des travaux de Brown, Michon, Peyrière [5].

Théorème 2.4. Soit $m$ une mesure de probabilité sur $[0,1[$ quasi-Bernoulli au sens faible. Alors $m$ vérifie le formalisme multifractal au sens suivant :

$$
\forall \alpha \in\left(-\tau^{\prime}(+\infty),-\tau_{d}^{\prime}(0)\right), \quad \operatorname{dim}\left(E_{\alpha}\right)=\operatorname{Dim}\left(E_{\alpha}\right)=\tau^{*}(\alpha)
$$

\section{Une large classe de mesures autosimilaires quasi-Bernoulli au sens faible}

\subsection{Présentation et énoncé du résultat principal}

On considère $N$ similitudes $S_{i}:[0,1] \rightarrow[0,1]$ de rapport de contraction respectif $\ell^{-\alpha_{i}}$ où $\ell$ est un entier supérieur ou égal à deux et pour tout $1 \leqslant i \leqslant N, \alpha_{i} \in \mathbb{N}^{*}$. Autrement dit,

$$
\forall 1 \leqslant i \leqslant N, \forall x \in[0,1], \quad S_{i}(x)=\ell^{-\alpha_{i}} x+\beta_{i} .
$$

Pour tout $1 \leqslant i \leqslant N$ on note $A_{i}$ l'image de l'intervalle [0,1] sous $S_{i}$. On a donc $\left|A_{i}\right|=\ell^{-\alpha_{i}}$.

On suppose de plus que : 
(i) $A_{i}$ est une réunion d'intervalles $\ell$-adiques c'est à dire que l'on peut écrire

$$
A_{i}=\bigcup_{k} B_{k, i}
$$

où $B_{k, i}$ est un intervalle $\ell$-adique.

(ii)

$$
[0,1]=\bigcup_{i} S_{i}([0,1])
$$

Ainsi $[0,1]$ est l'unique compact invariant par le système de fonctions itérées composé des similitudes $S_{i}$. De plus, on peut noter que dans cette situation les images des similitudes ne sont pas toutes de même taille et peuvent se recouvrir les unes les autres.

On introduit $r, s \in \mathbb{N}^{*}$ tels que :

$\min \left|A_{i}\right|=\ell^{-r}$ (en fait $\left.r=\max \alpha_{i}\right)$.

$\min \left|B_{k, i}\right|=\ell^{-s}$ (en fait cela signifie que l'image de chaque similitude peut s'écrire comme réunion d'intervalles $\ell$-adiques de génération $s$ ).

On a trivialement $r \leqslant s$.

On s'intéresse à la mesure autosimilaire $\mu$ solution de l'équation suivante

$$
\mu=\sum_{i=1}^{N} p_{i} \mu \circ S_{i}^{-1},
$$

où pour tout $1 \leqslant i \leqslant N, p_{i}>0$ avec $\sum p_{i}=1$.

Tout au long de cette partie, on assimile tout intervalle $\ell$-adique $I=I_{\epsilon_{1} \ldots \epsilon_{n}}$ au mot $\epsilon_{1} \ldots \epsilon_{n}$ formé de lettres de l'alphabet $\{0, \ldots, \ell-1\}$. De même, on note indifféremment $\sigma$ le shift en base $\ell$ de $[0,1$ [ dans lui même et le shift sur l'alphabet $\{0, \ldots, \ell-1\}$ défini par :

$$
\begin{aligned}
& \sigma:\{0, \ldots, \ell-1\}^{\mathbb{N}} \rightarrow\{0, \ldots, \ell-1\}^{\mathbb{N}} \\
&\left(\epsilon_{n}\right)_{n \in \mathbb{N}} \rightarrow\left(\epsilon_{n+1}\right)_{n \in \mathbb{N}} .
\end{aligned}
$$

Afin d'étudier $\mu$, on introduit les mesures de probabilité $\mu_{K}$, où $K$ parcourt tous les mots de l'alphabet $\{0, \ldots, \ell-1\}$ de longueur comprise entre $s-r$ et $s-1$, définies par :

$$
\forall n, \forall I \in \mathcal{F}_{n}, \quad \mu_{K}(I)=\frac{\mu(K I)}{\mu(K)} .
$$

(L'unique mot de longueur 0 sera par convention l'intervalle $[0,1]$.)

On a alors le résultat suivant :

Théorème 3.1. La mesure $\mu$ définie par l'Éq. (3.1) est quasi-Bernoulli au sens faible.

Le Théorème 3.1 et les résultats obtenus lors de la partie précédente permettent d'obtenir directement l'énoncé suivant relatif à l'analyse multifractale de la mesure $\mu$.

Corollaire 3.1. La fontion $\tau$ associée à la mesure $\mu$ définie par l'Éq. (3.1) est dérivable sur $\mathbb{R}^{+}$et $\mu$ vérifie le formalisme multifractal au sens suivant :

$$
\forall \alpha \in\left(-\tau^{\prime}(+\infty),-\tau_{d}^{\prime}(0)\right), \quad \operatorname{dim}\left(E_{\alpha}\right)=\operatorname{Dim}\left(E_{\alpha}\right)=\tau^{*}(\alpha) .
$$

Remarque. Les similitudes considérées ici vérifient la condition de type fini introduite par Ngai et Wang dans [22] et reprise par Feng dans le cas particulier où tous les rapports de contraction des similitudes sont égaux. Ainsi, 
lorque les rapports de contraction coïncident, le Corollaire 3.1 peut être vu comme un cas particulier d'un récent résultat de Feng (voir [9]). Toutefois, il semble difficile d'adapter le raisonnement de Feng au cas où les rapports de contraction ne sont pas tous égaux. En particulier, comme mentionné dans l'introduction, le Lemme 3.2 de [9] essentiel dans la démarche de Feng, est en défaut dans cette situation. Cela peut par exemple être vu en considérant

$$
\mu=p_{0} \mu \circ S_{0}^{-1}+p_{1} \mu \circ S_{1}^{-1}+p_{2} \mu \circ S_{2}^{-1},
$$

où

$$
S_{0}(x)=\frac{x}{2}, \quad S_{1}(x)=\frac{x}{2}+\frac{1}{2}, \quad S_{2}(x)=\frac{x}{4} .
$$

Dans le codage mis en place par Feng, les intervalles $I_{1}=\left[0, \frac{1}{8}\left[, I_{2}=\left[0, \frac{1}{32}\left[\right.\right.\right.\right.$ et $I_{3}=\left[0, \frac{1}{128}\right.$ [ qui appartiennent respectivement aux générations 1,2 et 3 issues de ce codage ont les mêmes caractéristiques au niveau de leur longueur et de leur type de voisinage. En particulier, pour $i \in\{1,2,3\}$, si $S_{\sigma}([0,1]) \cap I_{i} \neq \emptyset$ pour une composition de similitudes $S_{\sigma}$ dont le rapport de contraction $\rho_{\sigma}$ vérifie $\left(\frac{1}{4}\right)^{i+1}<\rho_{\sigma} \leqslant\left(\frac{1}{4}\right)^{i}$ alors $S_{\sigma}(0)=0$. Ainsi, en vertu du Lemme 3.2 de [9], les rapports $\mu\left(I_{2}\right) / \mu\left(I_{1}\right)$ et $\mu\left(I_{3}\right) / \mu\left(I_{2}\right)$ devraient être égaux. On vérifie aisément que cela n'est pas vrai dès que tous les coefficients $p_{i}$ sont strictement positifs.

\subsection{Résultats préliminaires à la preuve du Théorème 3.1}

Dans la suite de cette partie, on note $\mathcal{F}$ l'ensemble des intervalles $\ell$-adiques dont la génération est comprise entre $s-r$ et $s-1$. Le Théorème 3.1 repose essentiellement sur le fait que pour tout $K \in \mathcal{F}$ et pour tout intervalle $\ell$-adique $I$, on va pouvoir en itérant l'equation fonctionnelle (3.1) régissant $\mu$ exprimer $\mu_{K}(I)$ à l'aide d'un produit de matrices.

Pour cela, on définit pour tout intervalle $\ell$-adique $I$, le vecteur colonne $V(I)$ par :

$$
V(I)=\left(\begin{array}{c}
\mu_{0^{s-r}}(I) \\
\mu_{0^{s-r-1}}(I) \\
\vdots \\
\mu_{(\ell-1)^{s-1}}(I)
\end{array}\right) .
$$

$V(I)$ contient les quantités $\mu_{K}(I)$ où $K$ parcourt tous les mots de l'alphabet $\{0, \ldots, \ell-1\}$ dont la longueur est comprise entre $s-r$ et $s-1$, rangés dans l'ordre lexicographique. Par exemple, $0^{s-r}$ désigne le mot de longueur $s-r$ formé de $(s-r)$ zéros. On note $|K|$ la longueur de $K$. Enfin, signalons que l'on ne considère pas les quantités $\mu_{K}(I)$ avec $|K|<s-r$ afin de préserver l'irréductibilité de la matrice $M$ qui apparaît dans le Lemme 3.2.

On montre tout d'abord que :

\section{Lemme 3.1.}

$$
V(I)=M_{I}\left(\begin{array}{c}
1 \\
\vdots \\
1
\end{array}\right)
$$

où $M_{I}$ est une matrice carrée à coefficients positifs que l'on précisera.

Preuve du Lemme 3.1. Précisons tout d'abord quelques notations. Pour tout intervalle $\ell$-adique $I=I_{\epsilon_{1} \ldots \epsilon_{n}}$ de génération $n$ et pour tout $\epsilon \in\{0, \ldots, \ell-1\}$, on note $\epsilon I=I_{\epsilon \epsilon_{1} \ldots \epsilon_{n}}$ et $I \epsilon=I_{\epsilon_{1} \ldots \epsilon_{n} \epsilon}$. En outre si $A$ et $B$ désignent des matrices de même taille et $C$ un réel, alors $A>C$ signifie que tous les coefficients de $A$ sont strictement supérieurs à $C$ et $A>B$ signifiera que $A-B>0$. On adopte le même type de conventions pour les symboles $<$, $\leqslant$ et $\geqslant$. Enfin, si deux éléments $K$ et $K^{\prime}$ de $\mathcal{F}$ sont rangés aux places $i$ et $j$ dans l'ordre lexicographique, on se permettra d'écrire $A_{i, j}=A_{K, K^{\prime}}$. 
On montre que pour tout $\epsilon \in\{0, \ldots, \ell-1\}$, il existe une matrice $M_{\epsilon}$ telle que :

$$
V(\epsilon I)=M_{\epsilon} V(I) .
$$

Par suite, en itérant (3.3), si $I=\epsilon_{1} \ldots \epsilon_{n}$ alors $M_{I}$ sera le produit $M_{\epsilon_{1}} \ldots M_{\epsilon_{n}}$.

Construisons $M_{\epsilon}$. Les lignes de $M_{\epsilon}$ permettant le calcul de $\mu_{K}(\epsilon I)$ où $|K|<s-1$ se remplissent facilement. En effet d'après (3.2),

$$
\mu_{K}(\epsilon I)=\frac{\mu(K \epsilon)}{\mu(K)} \mu_{K \epsilon}(I) .
$$

Pour tout $K^{\prime} \in \mathcal{F}$, on pose alors

$$
\left(M_{\epsilon}\right)_{K, K^{\prime}}= \begin{cases}\frac{\mu(K \epsilon)}{\mu(K)} & \text { si } K^{\prime}=K \epsilon, \\ 0 & \text { sinon. }\end{cases}
$$

Il suffit donc de montrer que l'on peut écrire $\mu_{K}(\epsilon I)$ où $|K|=s-1$ comme combinaison linéaire de $\mu_{K^{\prime}}(I)$ où $s-r \leqslant\left|K^{\prime}\right| \leqslant s-1$. Étant donnée Eq. (3.1) régissant $\mu$, on doit donc déterminer les images réciproques de $K \epsilon$ par les similitudes $S_{i}$, c'est à dire les ensembles $S_{i}^{-1}(K \epsilon)$. En effet, on a :

$$
\mu_{K}(\epsilon I)=\frac{1}{\mu(K)} \sum_{i=1}^{N} p_{i} \mu \circ S_{i}^{-1}(K \epsilon I) .
$$

$S_{i}([0,1])$ est une réunion de $\ell^{s-\alpha_{i}}$ intervalles $\ell$-adiques de génération $s$ consécutifs et $K \epsilon$ est aussi un intervalle $\ell$-adique de génération $s$. Ainsi, deux cas peuvent se produire : soit $S_{i}^{-1}(K \epsilon)$ est vide, soit $S_{i}^{-1}(K \epsilon)$ est un intervalle $\ell$-adique de génération $s-\alpha_{i} \geqslant s-r$ noté $K_{i}$. Dans ce cas, pour tout $I$, on a $\mu \circ S_{i}^{-1}(K \epsilon I)=\mu\left(K_{i} I\right)$. La formule (3.5) devient

$$
\mu_{K}(\epsilon I)=\sum_{i \in \Lambda} p_{i} \frac{\mu\left(K_{i}\right)}{\mu(K)} \mu_{K_{i}}(I),
$$

où $\Lambda$ est l'ensemble des indices $i$ tels que $S_{i}^{-1}(K \epsilon)$ est non vide. Remarquons enfin que deux similitudes différentes génèrent des $K_{i}$ différents. Pour tout $K^{\prime} \in \mathcal{F}$, on pose alors :

$$
\left(M_{\epsilon}\right)_{K, K^{\prime}}=\left\{\begin{array}{ll}
p_{i} \frac{\mu\left(K^{\prime}\right)}{\mu(K)} & \text { si } \exists i, \\
0 & \text { sinon. }
\end{array} \quad K^{\prime}=S_{i}^{-1}(K \epsilon),\right.
$$

Ainsi, la matrice $M_{\epsilon}$ construite à partir des relations (3.4) et (3.6) est telle que (3.3) soit vérifiée. Ceci termine la preuve du Lemme 3.1.

Remarque. On a donc obtenu $\ell$ matrices $M_{0}, \ldots, M_{\ell-1}$ à coefficients positifs telles que $M=\sum_{i=0}^{\ell-1} M_{i}$ soit une matrice stochastique. (M stochastique provient simplement du fait que $\mu$ et les $\mu_{K}$ sont des mesures de probabilité.)

Lemme 3.2. $M$ est irréductible (c'est à dire $\forall(i, j), \exists k, M_{i, j}^{k}>0$ où $M_{i, j}^{k}$ désigne le terme situé à l'intersection la ième ligne et de la jème colonne de la matrice $\left.M^{k}\right)$.

Preuve du Lemme 3.2. On assimile $M$ à la matrice d'une chaîne de Markov dont les différents états sont représentés par l'ensemble $\mathcal{F}$ des intervalles $\ell$-adiques de génération comprise entre $s-r$ et $s-1$. On dira que $K$ communique directement avec $K^{\prime}$ si $M_{K, K^{\prime}}>0$ et que $K$ communique avec $K^{\prime}$ s'il existe $k$ tel que $M_{K, K^{\prime}}^{k}>0$ et on notera alors $K \rightarrow K^{\prime}$.

En utilisant les relations (3.4) et (3.6), on constate tout d'abord que la matrice $M=\sum_{\epsilon=0}^{\ell-1} M_{\epsilon}$ vérifie :

$$
\begin{cases}\operatorname{si}|K|<s-1, \quad M_{K, K^{\prime}}>0 & \Leftrightarrow \quad \exists \epsilon, \quad K^{\prime}=K \epsilon, \\ \text { si }|K|=s-1, \quad M_{K, K^{\prime}}>0 & \Leftrightarrow \quad \exists \epsilon, \exists i, \quad K^{\prime}=S_{i}^{-1}(K \epsilon) .\end{cases}
$$


Posons $J=0^{s-r}$. Pour montrer que $M$ est irréductible, on va montrer que $J$ communique avec chaque état $J^{\prime}$ vérifiant $s-r \leqslant\left|J^{\prime}\right| \leqslant s-1$ et qu'inversement tout état $J^{\prime}$ communique avec $J$.

On introduit le sous arbre en base $\ell$ qui part de la génération $s-r$ et qui s'arrête à la génération $s-1$.

Notons que si $J^{\prime}$ est un mot de longueur strictement inférieure à $s-1$, on déduit de (3.7) que $J^{\prime}$ communique directement avec ses fils. Par suite, $J^{\prime}$ communique avec tous ses descendants.

Etape 1. Montrons que $J=0^{s-r}$ communique avec tous les états $J^{\prime}$. Comme tout état de génération strictement supérieure à $s-r$ peut se voir comme un descendant d'un état de génération $s-r$, il suffit d'après la remarque précédente de montrer que $J$ communique avec tous les éléments $J^{\prime}$ de génération $s-r$. Ainsi, fixons un élément $J^{\prime}$ de génération $s-r$.

Considérons une similitude $S_{i_{0}}$ ayant pour image $S_{i_{0}}([0,1])=\left[0, \ell^{-\alpha_{i_{0}}}\right]$, ce qui est possible car par hypothèse $[0,1]=\bigcup S_{i}([0,1])$. Deux cas sont alors possibles :

(i) Soit $\alpha_{i_{0}}=s$, auquel cas $\alpha_{i_{0}}=r=s$, et $J=0^{s-r}$ est donc le seul état de génération $s-r$. On a donc $J^{\prime}=J$. En outre, si $L=0^{s-1}$, on a

$$
S_{i_{0}}^{-1}(L 0)=S_{i_{0}}^{-1}\left(0^{\alpha_{i_{0}}}\right)=[0,1]=J .
$$

D'après (3.7), on déduit que $L \rightarrow J$. Comme, $J \rightarrow L$ par descendance, on trouve que $J \rightarrow J$, ce qui termine pour ce cas la preuve de l'étape 1 .

(ii) Soit $\alpha_{i_{0}}<s$. Montrons tout d'abord que $L=0^{s-1}$ communique avec tous les éléments de sa génération. Pour cela, on remarque que $S_{i_{0}}([0,1])$ est réunion de $\ell^{s-\alpha_{i_{0}}}$ intervalles $\ell$-adiques de génération $s$. Ces intervalles se codent sous la forme $K \epsilon$ où $K$ parcourt l'ensemble des $\ell^{s-1-\alpha_{i_{0}}}$ premiers mots de génération $s-1$ et $\epsilon \epsilon$ $\{0, \ldots, \ell-1\}$. En fixant $K$ et en faisant varier $\epsilon$, la relation (3.7) nous dit alors que chacun des $\ell^{s-1-\alpha_{i}}$ premiers éléments de génération $s-1$ communique avec $\ell$ éléments consécutifs de génération $s-\alpha_{i_{0}}$ de la manière suivante : le premier élément de la génération $s-1$ communique avec les $\ell$ premiers éléments de la génération $s-\alpha_{i_{0}}$, le deuxième élément de la génération $s-1$ communique avec les $\ell$ éléments suivants de la génération $s-\alpha_{i_{0}} \ldots$.

En particulier $L=0^{s-1}$ communique avec les $\ell$ premiers mots de génération $s-\alpha_{i_{0}}$, puis par descendance, il communique avec les $\ell^{\alpha_{i}}$ premiers éléments de sa génération. Deux cas peuvent alors se produire. Soit $\alpha_{i_{0}}=s-1$, auquel cas on a épuisé par cette opération tous les éléments de la génération $s-1$. Soit $\alpha_{i_{0}}<s-1$. On peut alors recommencer le procédé avec le deuxième élément de la génération $s-1$ (à savoir $0^{s-2} 1$ ) et on montre que $L$ communique aussi avec les éléments de génération $s-1$ classés entre les places $\ell^{\alpha_{i}}+1$ et $2 \ell^{\alpha_{i}}$. De proche en proche, on obtient que $L$ communique avec tous les éléments de sa génération.

Enfin, on considère une similitude $S_{j_{0}}$ ayant pour rapport de contraction $\ell^{-r} . J^{\prime}$ est alors l'image inverse par $S_{j_{0}}$ d'un intervalle de génération $s$. Il existe donc un élément $L^{\prime}$ de génération $s-1$ et $\epsilon \in\{0, \ldots, \ell-1\}$ tels que

$$
J^{\prime}=S_{j_{0}}^{-1}\left(L^{\prime} \epsilon\right)
$$

Ainsi, d'après (3.7), $L^{\prime} \rightarrow J^{\prime}$.

On trouve finalement que $J \rightarrow L \rightarrow L^{\prime} \rightarrow J^{\prime}$, ce qui termine la preuve de ce deuxième cas et celle de l'étape 1 .

Etape 2. Montrons que tout état $J^{\prime} \in \mathcal{F}$ communique avec $J=0^{s-r}$. Un état communiquant avec ses descendants, il suffit de considérer un état $J^{\prime}$ de génération $s-1$. Au cours de la preuve de l'étape 1 , on a vu entre autres que $L=0^{s-1}$ communique avec tous les éléments de la génération $s-r$. En particulier, $L \rightarrow J$. Ainsi, il suffit de montrer que $J^{\prime} \rightarrow L$. Pour cela, on va reprendre l'argument utilisé dans la preuve de l'étape 1 montrant que $L=0^{s-1}$ communique avec tous les éléments de sa génération.

Rappelons que $J^{\prime}$ est de génération $s-1$. Ainsi, $J^{\prime}$ est réunion de ses fils $J^{\prime} \epsilon$ qui sont de génération $s$. Par ailleurs, pour tout $i, S_{i}([0,1])$ est lui même réunion d'intervalles de génération $s$. Là encore, deux cas sont possibles :

(i) Soit on peut trouver une similitude $S_{i_{0}}$ et un fils $J^{\prime} \in$ qui soit le premier intervalle intervenant dans le découpage de $S_{i_{0}}([0,1])$. Dans ce cas,

$$
S_{i_{0}}^{-1}\left(J^{\prime} \epsilon\right)=0^{s-\alpha_{i_{0}}} .
$$


Par suite, d'après (3.7), $J^{\prime} \rightarrow 0^{s-\alpha_{i}}$. Par descendance, on déduit que $J^{\prime}$ communique avec $L=0^{s-1}$, ce qui termine la preuve de l'étape 2 dans ce cas.

(ii) Soit on n'est pas dans le cas précédent. Comme $[0,1]=\bigcup S_{i}([0,1])$, il est facile de voir qu'alors on peut trouver une similitude $S_{i_{0}}$ telle que $J^{\prime} \subset S_{i_{0}}([0,1])$. Ainsi, pour tout $\epsilon$, les ensembles $S_{i_{0}}^{-1}\left(J^{\prime} \epsilon\right)$ sont non vides. On déduit alors de (3.7) que $J^{\prime}$ communique avec $\ell$ éléments consécutifs de la génération $s-\alpha_{i_{0}}$. Par descendance, on déduit que $J^{\prime}$ communique avec $\ell^{\alpha_{i_{0}}}$ éléments consécutifs $J_{0}^{\prime}, \ldots, J_{\ell^{\alpha_{i}-1}}^{\prime}$ de sa génération. Les intervalles $J_{0}^{\prime}, \ldots, J_{\ell^{\alpha_{i}-1}}^{\prime}$ forment un agglomérat de $\ell^{\alpha_{i}}$ intervalles de génération $s-1$ contigus.

Deux cas peuvent à nouveau se produire. Soit l'un des $J_{j}^{\prime}$ vérifie la condition (i) et on conclut que $J^{\prime} \rightarrow J_{j}^{\prime} \rightarrow L$, ce qui termine la preuve. Sinon, c'est qu'il existe une similitude $S_{i_{1}}$ telle que

$$
\bigcup_{j} J_{j}^{\prime} \subset S_{i_{1}}([0,1])
$$

On peut recommencer la même opération avec cette nouvelle similitude et construire ainsi un agglomérat de $\ell^{\alpha_{i}+\alpha_{i_{1}}}$ intervalles de génération $s-1$ tel que $J^{\prime}$ communique avec chaque élément de cet agglomérat.

En itérant ce raisonnement, on arrive forcément au bout d'un certain temps à avoir l'alternative $(i)$ et à conclure que $J^{\prime} \rightarrow L$. Ceci achève la preuve de l'étape 2 et du Lemme 3.2.

Le lemme 3.2 fournit alors des renseignements sur les propriétés d'homogénéité des mesures $\mu_{K}$ localisées en $K$ définies par la relation (3.2) où $K$ parcourt tous les mots de l'alphabet $\{0, \ldots, \ell-1\}$ de longueur comprise entre $s-r$ et $s-1$. Cela est l'objet de l'énoncé suivant :

Lemme 3.3. Pour tout $K \in \mathcal{F}$, la mesure $\mu_{K}$ définie par la relation (3.2) est quasi-Bernoulli au sens faible.

Preuve du Lemme 3.3. Traitons par exemple le cas $K=0^{s-r}$, les autres se traitant de manière similaire. D'après le Lemme 3.1, $\mu_{K}(I)$ est la somme des éléments de la première ligne de $M_{I}$. Dans la suite, on note

$$
\mu_{K}(I)=\left\|M_{I}\right\| .
$$

De plus, on désigne par $\mathcal{I}$ la matrice identité de même taille que $M$ et par $E$ la matrice, de même taille que $M$, dont tous les éléments de la première colonne sont égaux à 1 et tous les autres coefficients sont nuls. Remarquons que $\|A E B\|=\|A\|\|B\|$ pour toute matrice $A$ et $B$ de la même taille que $M$.

D'après le Lemme 3.2, $M$ est irréductible. Il existe donc un entier $m \in \mathbb{N}^{*}$ tel que $\sum_{k=1}^{m} M^{k}>0$. On peut donc trouver une constante $C>0$ telle que

$$
E \sum_{k=1}^{m} M^{k} \geqslant C \quad \text { et } \quad E \leqslant C \sum_{k=1}^{m} M^{k}
$$

Par suite, pour tout $I \in \mathcal{F}_{n}$ et pour tout intervalle $\ell$-adique $J$, on a :

$$
\begin{aligned}
\mu_{K}(I J) & =\left\|M_{I J}\right\|=\left\|M_{I} M_{J}\right\|=\left\|M_{I} \mathcal{I} M_{J}\right\| \leqslant \frac{1}{C}\left\|M_{I} E\left(\sum_{k=1}^{m} M^{k}\right) M_{J}\right\|=\frac{1}{C}\left\|M_{I}\right\|\left\|\sum_{k=1}^{m} M^{k} M_{J}\right\| \\
& =\frac{1}{C} \mu_{K}(I) \sum_{k=1}^{m}\left\|M^{k} M_{J}\right\|=\frac{1}{C} \mu_{K}(I) \sum_{k=1}^{m} \mu_{K}\left(\sigma^{-k}(J)\right) .
\end{aligned}
$$

On obtient de manière similaire :

$$
\mu_{K}(I) \mu_{K}(J)=\left\|M_{I}\right\|\left\|M_{J}\right\|=\left\|M_{I} E M_{J}\right\| \leqslant C\left\|M_{I}\left(\sum_{k=1}^{m} M^{k}\right) M_{J}\right\|
$$




$$
=C \sum_{k=1}^{m}\left\|M_{I} M^{k} M_{J}\right\|=C \sum_{k=1}^{m} \mu_{K}\left(I \cap \sigma^{-(n+k)}(J)\right) .
$$

Ceci montre que $\mu_{K}$ est quasi-Bernoulli au sens faible et prouve le Lemme 3.3.

Avant d'en venir à la preuve du Théorème 3.1, on énonce un dernier résultat qui permet de comparer la mesure $\mu$ et les mesures localisées $\mu_{K}$ pour lesquelles on vient de montrer le caractère quasi-Bernoulli au sens faible.

Lemme 3.4. Pour tout $K \in \mathcal{F}$, il existe un entier $t_{K} \in \mathbb{N}$ et une constante $C(K)>0$ tels que

$$
\mu \leqslant C(K) \mu_{K} \circ \sigma^{-t_{K}} .
$$

Preuve du Lemme 3.4. Comme,

$$
[0,1]=\bigcup_{i} S_{i}([0,1])
$$

il existe une similitude $S_{i_{0}}$ dont l'image est de la forme $\left[0, \ell^{-\alpha_{i_{0}}}\right]$ avec $\alpha_{i_{0}} \leqslant r$. Il existe alors un entier $n \in \mathbb{N}$ tel que

$$
s-r \leqslant n \alpha_{i_{0}} \leqslant s-1
$$

En outre, pour tout intervalle $\ell$-adique $I$, on a en utilisant l'Éq. (3.1) régissant $\mu$,

$$
\mu\left(0^{n \alpha_{i}} I\right) \geqslant\left(p_{i_{0}}\right)^{n} \mu(I)
$$

Par suite, $\mu \leqslant C \mu_{K^{\prime}}$ où $K^{\prime}=0^{n \alpha_{i}}$. De plus, l'irréductibilité de $M$ entraîne que pour tout $K \in \mathcal{F}$, il existe un entier $t_{K} \in \mathbb{N}$ tel que $M_{K, K^{\prime}}^{t_{K}}>0$ ou encore

$$
\left(\sum_{i=0}^{\ell-1} M_{i}\right)_{K, K^{\prime}}^{t_{K}}>0
$$

Il existe donc $\epsilon_{1}, \ldots, \epsilon_{t_{K}} \in\{0, \ldots, \ell-1\}^{t_{K}}$ tels que $\left(M_{\epsilon_{1}} \cdots M_{\epsilon_{t_{K}}}\right)_{K, K^{\prime}}>0$. Comme, pour tout intervalle $\ell$-adique $I$,

$$
\mu_{K}\left(\epsilon_{1} \cdots \epsilon_{t_{K}} I\right) \geqslant\left(M_{\epsilon_{1}} \cdots M_{\epsilon_{t_{K}}}\right)_{K, K^{\prime}} \mu_{K^{\prime}}(I),
$$

on déduit finalement que pour tout $K \in \mathcal{F}$, il existe un entier $t_{K} \in \mathbb{N}$ et une constante $C(K)>0$ tels que

$$
\mu \leqslant C \mu_{K^{\prime}} \leqslant C(K) \mu_{K} \circ \sigma^{-t_{K}},
$$

ce qui prouve le Lemme 3.4.

\subsection{Preuve du Théorème 3.1}

La preuve du Théorème 3.1 s'appuie sur procédé déjà utilisé qui permet de modifier les inégalités qui apparaissent dans la définition des mesures quasi-Bernoulli au sens faible (voir (1.3)) en augmentant les entiers de 1 (voir (2.8)).

Etape 1. Montrons qu'il existe des entiers $p, s_{1}, s_{2}$ tels que pour tout couple $(n, q)$ et pour tout intervalle $\ell$-adique $I \in \mathcal{F}_{n}, J \in \mathcal{F}_{q}$, on a

$$
\mu\left(I \cap \sigma^{-(n+p)}(J)\right) \leqslant C \mu(I) \sum_{s=s_{1}}^{s_{2}} \mu\left(\sigma^{-s}(J)\right),
$$

où $C$ est une constante positive indépendante de $n, q, I$ et $J$. 
On suppose tout d'abord $n \geqslant s-r$. Il existe alors $K \in \mathcal{F}_{s-r}$ tel que $I$ s'écrive $I=K I^{\prime}$. De plus, d'après le Lemme 3.3, $\mu_{K}$ est quasi-Bernoulli au sens faible. Il existe donc des entiers $p_{K}, s_{1}(K), s_{2}(K)$ indépendants de $I^{\prime}$ et $J$ tels que

$$
\mu_{K}\left(I^{\prime} \cap \sigma^{-\left(n^{\prime}+p_{K}\right)}(J)\right) \leqslant C(K) \mu_{K}\left(I^{\prime}\right) \sum_{s=s_{1}(K)}^{s_{2}(K)} \mu_{K}\left(\sigma^{-s}(J)\right),
$$

où $n^{\prime}=\left|I^{\prime}\right|=n-(s-r)$ et $C(K)$ est une constante qui ne dépend que de $K$. Par suite, en notant indifféremment $C(K)$ toute constante dépendant uniquement de $K$, on a

$$
\mu\left(I \cap \sigma^{-\left(n+p_{K}\right)}(J)\right)=C(K) \mu_{K}\left(I^{\prime} \cap \sigma^{-\left(n^{\prime}+p_{K}\right)}(J)\right) \leqslant C(K) \mu_{K}\left(I^{\prime}\right) \sum_{s=s_{1}(K)}^{s_{2}(K)} \mu_{K}\left(\sigma^{-s}(J)\right),
$$

ce qui donne finalement pour tout intervalle $I$ de la forme $I=K I^{\prime}$,

$$
\mu\left(I \cap \sigma^{-\left(n+p_{K}\right)}(J)\right) \leqslant C(K) \mu(I) \sum_{s=s_{1}(K)+s-r}^{s_{2}(K)+s-r} \mu\left(\sigma^{-s}(J)\right) .
$$

Par conséquent, en considérant l'ensemble des intervalles $K$ de la génération $s-r$ et en utilisant le procédé (voir (2.8)) évoqué ci-dessus pour rendre tous les $p_{K}$ égaux, on peut donc trouver des entiers $p^{\prime}$, $s_{1}^{\prime}$ et $s_{2}^{\prime}$ tels que pour tout intervalle $\ell$-adique $I$ de génération supérieure ou égale à $s-r$ et tout intervalle $\ell$-adique $J$, on ait :

$$
\mu\left(I \cap \sigma^{-\left(n+p^{\prime}\right)}(J)\right) \leqslant C \mu(I) \sum_{s=s_{1}^{\prime}}^{s_{2}^{\prime}} \mu\left(\sigma^{-s}(J)\right) .
$$

(On peut par exemple prendre

$$
\left.p^{\prime}=\max _{K} p_{K}, \quad s_{1}^{\prime}=\min _{K} s_{1}(K)+s-r \quad \text { et } \quad s_{2}^{\prime}=\max _{K} s_{2}(K)+\max _{K} p_{K}-\min _{K} p_{K}+s-r .\right)
$$

Finalement, en prenant $p=p^{\prime}+s-r$, on peut trouver des entiers $s_{1}$ et $s_{2}$ tels que pour tout intervalle $\ell$-adique $I \in \mathcal{F}_{n}$ et $J \in \mathcal{F}_{q}$, l'inégalité (3.8) soit vérifiée. En effet, si $n \geqslant s-r$, il suffit d'appliquer le procédé (2.8) à la relation (3.9) pour transformer $p^{\prime}$ en $p$. Si $n<s-r$, la relation (3.9) montre que :

$$
\begin{aligned}
\mu\left(I \cap \sigma^{-(n+p)}(J)\right) & =\sum_{L \in \mathcal{F}_{s-r}} \mu\left(I L \cap \sigma^{-\left(n+p^{\prime}+s-r\right)}(J)\right) \leqslant C \sum_{L \in \mathcal{F}_{s-r}} \mu(I L)\left(\sum_{s=s_{1}^{\prime}}^{s_{2}^{\prime}} \mu\left(\sigma^{-s}(J)\right)\right) \\
& =C \mu(I) \sum_{s=s_{1}^{\prime}}^{s_{2}^{\prime}} \mu\left(\sigma^{-s}(J)\right) .
\end{aligned}
$$

Le regroupement des cas $n \geqslant s-r$ et $n<s-r$ entraîne alors facilement la relation (3.8) et termine la preuve de l'étape 1.

Etape 2. Montrons qu'il existe des entiers $r, p_{1}, p_{2}$ tels que pour tout couple $(n, q)$ et pour tout intervalle $\ell$-adique $I \in \mathcal{F}_{n}, J \in \mathcal{F}_{q}$, on a

$$
C \mu(I) \mu\left(\sigma^{-r}(J)\right) \leqslant \sum_{k=p_{1}}^{p_{2}} \mu\left(I \cap \sigma^{-(n+k)}(J)\right),
$$

où $C$ est une constante positive indépendante de $n, q, I$ et $J$. 
On suppose tout d'abord $n \geqslant s-r$. Il existe alors $K \in \mathcal{F}_{s-r}$ tel que $I$ s'écrive $I=K I^{\prime}$. De plus, d'après le Lemme 3.3, $\mu_{K}$ est quasi-Bernoulli au sens faible. Il existe donc des entiers $r_{K}, p_{1}(K), p_{2}(K)$ indépendants de $I^{\prime}$ et $J$ tels que

$$
C(K) \mu_{K}\left(I^{\prime}\right) \mu_{K}\left(\sigma^{-r_{K}}(J)\right) \leqslant \sum_{k=p_{1}(K)}^{p_{2}(K)} \mu_{K}\left(I^{\prime} \cap \sigma^{-\left(n^{\prime}+k\right)}(J)\right),
$$

où $n^{\prime}=n-(s-r)$. Par ailleurs, d'après le Lemme 3.4, il existe un entier $t_{K} \in \mathbb{N}$ tel que

$$
\mu \leqslant C(K) \mu_{K} \circ \sigma^{-t_{K}} .
$$

Ainsi, quitte à remplacer $C(K)$ par $C(K) \mu(K)$, on a

$$
\mu(I) \mu\left(\sigma^{-r_{K}}(J)\right) \leqslant C(K) \mu_{K}\left(I^{\prime}\right) \mu_{K}\left(\sigma^{-\left(r_{K}+t_{K}\right)}(J)\right)
$$

ce qui d'après la relation (3.11) donne en utilisant un argument de classe monotone,

$$
\mu(I) \mu\left(\sigma^{-r_{K}}(J)\right) \leqslant C(K) \sum_{k=p_{1}(K)}^{p_{2}(K)} \mu_{K}\left(I^{\prime} \cap \sigma^{-\left(n^{\prime}+k\right)}\left(\sigma^{-t_{K}}(J)\right)\right) .
$$

On peut alors trouver des entiers $P_{1}(K), P_{2}(K)$ indépendants de $I$ et $J$ tels que

$$
\mu(I) \mu\left(\sigma^{-r_{K}}(J)\right) \leqslant C(K) \sum_{k=P_{1}(K)}^{P_{2}(K)} \mu\left(I \cap \sigma^{-(n+k)}(J)\right) .
$$

En raisonnant comme à l'étape 1, on peut, en considérant l'ensemble des éléments $K \in \mathcal{F}_{s-r}$, trouver des entiers $r, p_{1}, p_{2}$ tels que (3.10) soit vérifiée.

Etape 3. Montrons que les relations (3.8) et (3.10) entraînent que $\mu$ est quasi-Bernoulli au sens faible. Deux cas sont alors possibles :

(i) Le cas $p \leqslant p_{1}$. Par le procédé (2.8), on transforme (3.8) pour modifier successivement $p$ en $p_{1}, p_{1}+$ $1, \ldots, p_{2}$. En sommant les inégalités obtenues, on peut alors trouver des entiers $s_{1}^{\prime}$ et $s_{2}^{\prime}$ tels que

$$
\frac{1}{C} \mu(I) \mu\left(\sigma^{-r}(J)\right) \leqslant \sum_{k=p_{1}}^{p_{2}} \mu\left(I \cap \sigma^{-(n+k)}(J)\right) \leqslant C \sum_{s=s_{1}^{\prime}}^{s_{2}^{\prime}} \mu\left(\sigma^{-s}(J)\right),
$$

ce qui termine la preuve du Théorème 3.1 dans cette situation.

(ii) Le cas $p>p_{1}$. Toujours grâce au procédé (2.8), on transforme (3.10) pour modifier $p_{1}$ en $p$ et on se ramène alors au cas précédent.

Le regroupement des cas (i) et (ii) montre que $\mu$ est quasi-Bernoulli au sens faible et termine la preuve du Théorème 3.1.

\subsection{Exemple 1}

On fixe un entier $b$ strictement positif et on considère la mesure de probabilité $\mu$ qui vérifie l'équation suivante

$$
\mu=p_{0} \mu \circ S_{0}^{-1}+p_{1} \mu \circ S_{1}^{-1}+p_{2} \mu \circ S_{2}^{-1},
$$

avec

$$
S_{0}(x)=\frac{x}{2}, \quad S_{1}(x)=\frac{x}{2^{b}}+\frac{2^{b}-1}{2^{b+1}}, \quad S_{2}(x)=\frac{x}{2}+\frac{1}{2} .
$$

La mesure $\mu$ entre dans le cadre que l'on vient d'étudier avec $\ell=2$ et est donc d'après le Théorème 3.1 quasiBernoulli au sens faible. 
Remarque 1. En général, $\mu$ n'est pas quasi-Bernoulli. Traitons par exemple le cas $p_{1}>p_{0}^{b}$. Si on note $u_{n}=$ $\mu\left(10^{n b}\right)$, alors d'après l'équation fonctionnelle (3.12) vérifiée par $\mu$, on a

$$
u_{n}=p_{2} \mu(0) p_{0}^{n b-1}+p_{1} u_{n-1} \text {. }
$$

En itérant cette relation on obtient :

$$
u_{n}=p_{2} \mu(0) p_{0}^{n b-1} \sum_{k=0}^{n-1}\left(\frac{p_{1}}{p_{0}^{b}}\right)^{k}+p_{1}^{n} u_{0} .
$$

De cela, on déduit facilement que $u_{n}$ est de l'ordre de $p_{1}^{n}$. Or si $\mu$ était quasi-Bernoulli, alors asymptotiquement $u_{n}$ serait de l'ordre de $\mu\left(0^{n b}\right)$, lequel par (3.12) équivaut à $p_{0}^{n b}$.

Remarque 2. Si $b=1$, les similitudes définissant la mesure $\mu$ entrent dans le cadre d'utilisation du résultat de Feng (voir [9]) et ainsi une partie de son analyse multifractale peut aussi se déduire de [9]. Ce n'est plus le cas lorsque $b>1$. Enfin, on verra dans la partie 4.3 comment dans le cas $b=1$ la mesure $\mu$ peut être utilisée dans un contexte autoaffine déjà étudié par Kenyon et Peres dans [16].

Pour familiariser le lecteur à notre démarche, terminons cette partie par la construction de la matrice $M$ dans le cas où $b=2$. Dans cette situation, comme $s-r=1$ et $s-1=2$, la matrice $M=M_{0}+M_{1}$ est de taille 6. Soit $I$ un intervalle dyadique. Des relations,

$$
\begin{aligned}
& \mu(000 I)=p_{0} \mu(00 I), \quad \mu(001 I)=p_{0} \mu(01 I), \quad \mu(010 I)=p_{0} \mu(10 I), \\
& \mu(011 I)=p_{0} \mu(11 I)+p_{1} \mu(0 I), \quad \mu(100 I)=p_{2} \mu(00 I)+p_{1} \mu(1 I), \quad \mu(101 I)=p_{2} \mu(01 I), \\
& \mu(110 I)=p_{2} \mu(10 I), \quad \mu(111 I)=p_{2} \mu(11 I),
\end{aligned}
$$

et du calcul des masses des intervalles des deux premières générations, on déduit que

$$
\begin{aligned}
& M_{0}=\left(\begin{array}{cccccc}
0 & 0 & p_{0} & 0 & 0 & 0 \\
0 & 0 & 0 & 0 & p_{0}+p_{1} & 0 \\
0 & 0 & p_{0} & 0 & 0 & 0 \\
0 & 0 & 0 & 0 & \frac{p_{2}\left(p_{0}+p_{1}\right)}{p_{1}+p_{2}} & 0 \\
0 & \frac{p_{1}}{p_{0}+p_{1}} & \frac{p_{0}^{2}}{p_{0}+p_{1}} & 0 & 0 & 0 \\
0 & 0 & 0 & 0 & p_{0}+p_{1} & 0
\end{array}\right), \\
& M_{1}=\left(\begin{array}{cccccc}
0 & 0 & 0 & p_{1}+p_{2} & 0 & 0 \\
0 & 0 & 0 & 0 & 0 & p_{2} \\
0 & 0 & 0 & p_{1}+p_{2} & 0 & 0 \\
\frac{p_{1}}{p_{1}+p_{2}} & 0 & 0 & 0 & 0 & \frac{p_{2}^{2}}{p_{1}+p_{2}} \\
0 & 0 & 0 & \frac{p_{0}\left(p_{1}+p_{2}\right)}{p_{0}+p_{1}} & 0 & 0 \\
0 & 0 & 0 & 0 & 0 & p_{2}
\end{array}\right)
\end{aligned}
$$

et donc

$$
M=\left(\begin{array}{cccccc}
0 & 0 & p_{0} & p_{1}+p_{2} & 0 & 0 \\
0 & 0 & 0 & 0 & p_{0}+p_{1} & p_{2} \\
0 & 0 & p_{0} & p_{1}+p_{2} & 0 & 0 \\
\frac{p_{1}}{p_{1}+p_{2}} & 0 & 0 & 0 & \frac{p_{2}\left(p_{0}+p_{1}\right)}{p_{1}+p_{2}} & \frac{p_{2}^{2}}{p_{1}+p_{2}} \\
0 & \frac{p_{1}}{p_{0}+p_{1}} & \frac{p_{0}^{2}}{p_{0}+p_{1}} & \frac{p_{0}\left(p_{1}+p_{2}\right)}{p_{0}+p_{1}} & 0 & 0 \\
0 & 0 & 0 & 0 & p_{0}+p_{1} & p_{2}
\end{array}\right) .
$$

On vérifie aisément que $M$ est irréductible. 


\subsection{Une généralisation}

On peut étendre les résultats obtenus et en particulier le Théorème 3.1 en s'autorisant non seulement des similitudes de rapport positif mais aussi des similitudes de rapport négatif. On obtient ainsi l'énoncé plus général suivant :

Théorème 3.2. On considère $N$ similitudes $S_{i}:[0,1] \rightarrow[0,1]$ s'écrivant $S_{i}(x)=\epsilon_{i} \ell^{-\alpha_{i}} x+\beta_{i}$ avec $\alpha_{i} \in \mathbb{N}^{*}$, $\epsilon_{i} \in\{ \pm 1\}$ dont les images sont des réunions d'intervalles $\ell$-adiques. On suppose enfin que $[0,1]=\bigcup S_{i}([0,1])$. Alors l'unique mesure de probabilité $\mu$ sur $[0,1]$ vérifiant :

$$
\mu=\sum_{i=1}^{N} p_{i} \mu \circ S_{i}^{-1}
$$

est quasi-Bernoulli au sens faible. Par suite, $\tau$ est dérivable sur $\mathbb{R}^{+}$et $\mu$ vérifie le formalisme multifractal au sens suivant :

$$
\forall \alpha \in\left(-\tau^{\prime}(+\infty),-\tau_{d}^{\prime}(0)\right), \quad \operatorname{dim}\left(E_{\alpha}\right)=\operatorname{Dim}\left(E_{\alpha}\right)=\tau^{*}(\alpha) .
$$

Remarque. Dans [28], on montre que contrairement à la situation quasi-Bernoulli, la fonction $\tau$ associée à la mesure $\mu$ peut présenter plusieurs points de non dérivabilité sur $\mathbb{R}^{-}$. De plus, pour les valeurs de $\alpha$ se situant dans une transition de phase, la relation $\operatorname{dim}\left(E_{\alpha}\right)=\tau^{*}(\alpha)$ n'est pas toujours vérifiée.

Idée de la preuve du Théorème 3.2. La preuve, reprenant les mêmes idées que la preuve Théorème 3.1, on se contentera de mentionner la différence principale. Dans ce contexte, afin de tenir compte des changements de signe, on est amené à considérer les mesures $\mu_{K}$ et les mesures $(\mu \circ T)_{K}$ où $T$ est la symétrie par rapport au point $\frac{1}{2}$ allant de l'intervalle $[0,1]$ dans lui même. Pour tout intervalle $\ell$-adique $I$, le nouveau vecteur $V(I)$ s'écrit donc :

$$
V(I)=\left(\begin{array}{c}
\mu_{0^{s-r}}(I) \\
(\mu \circ T)_{0^{s-r}}(I) \\
\mu_{0^{s-r-1}}(I) \\
(\mu \circ T)_{0^{s-r-1}}(I) \\
\vdots \\
\mu_{(\ell-1)^{s-1}}(I) \\
(\mu \circ T)_{(\ell-1)^{s-1}}(I)
\end{array}\right) .
$$

Comme précédemment, on montre que l'on peut exprimer $V(I)$ comme un produit de matrices. On construit ainsi une matrice $M$ irréductible qui permet de montrer que toutes les mesures composant les lignes de $V(I)$ sont quasiBernoulli au sens faible. Le reste de la preuve du Théorème 3.2 ne présente pas de changement majeur par rapport à celle du Théorème 3.1.

\subsection{Exemple 2}

On se propose ici d'étudier la famille de mesures autosimilaires solutions de l'équation suivante

$$
\mu=\sum_{i=0}^{3} p_{i} \mu \circ S_{i}^{-1}
$$

où

$$
S_{0}(x)=\frac{x}{2}, \quad S_{1}(x)=\frac{x}{2}+\frac{1}{2}, \quad S_{2}(x)=-\frac{x}{2}+\frac{1}{2}, \quad S_{3}(x)=-\frac{x}{2}+1 \quad \text { et } \quad \sum_{i=0}^{3} p_{i}=1 .
$$


Ces mesures entrent dans le contexte des mesures étudiées dans la partie 3.5 avec $\ell=2, r=s=1$. On montrera lors de la partie 4.2 comment ces mesures apparaissent dans un contexte autoaffine classique étudié par Urbański [30].

La proposition suivante met l'accent sur le caractère d'homogénéité de $\mu$ en fonction de la nullité de certains coefficients et précise ainsi le Théorème 3.2 dans ce cas particulier.

Proposition 3.1. La mesure $\mu$ définie par l'Éq. (3.13) est quasi-Bernoulli au sens faible. De façon plus précise, si $N=\#\left\{i \in\{0,1,2,3\}, p_{i}=0\right\}$ alors on a les cas suivants :

(i) Si $N=3$ alors $\mu$ est une masse de Dirac.

(ii) Si $N \in\{0,2\}$ alors $\mu$ est quasi-Bernoulli sur son support.

(iii) Si $N=1$ alors $\mu$ possède la propriété suivante :

$$
\left\{\begin{array}{l}
\exists C>0, \forall n, \forall p, \forall I \in \mathcal{F}_{n}, \forall J \in \mathcal{F}_{p}, \\
\frac{1}{C} \mu(I) \mu(J) \leqslant \mu\left(I \cap \sigma^{-(n+1)}(J)\right) \leqslant C \mu(I) \mu\left(\sigma^{-2}(J)\right) .
\end{array}\right.
$$

En reprenant les notations déjà introduites et en notant $I^{*}:=T^{-1}(I)$, on obtient en utilisant l'Éq. (3.13) les relations :

$$
\left\{\begin{array}{l}
\mu(0 I)=p_{0} \mu(I)+p_{2} \mu\left(I^{*}\right) \\
\mu(1 I)=p_{1} \mu(I)+p_{3} \mu\left(I^{*}\right)
\end{array}\right.
$$

Comme $\left(I^{*}\right)^{*}=I$, on déduit facilement la relation matricielle suivante

$$
\left(\begin{array}{c}
\mu(I) \\
\mu \circ T(I)
\end{array}\right)=\left(\begin{array}{c}
\mu(I) \\
\mu\left(I^{*}\right)
\end{array}\right)=M_{\epsilon_{1}} \ldots M_{\epsilon_{n}}\left(\begin{array}{l}
1 \\
1
\end{array}\right)
$$

où $I=I_{\epsilon_{1} \ldots \epsilon_{n}}$ et $M_{0}, M_{1}$ sont les matrices suivantes :

$$
M_{0}=\left(\begin{array}{ll}
p_{0} & p_{2} \\
p_{3} & p_{1}
\end{array}\right) \quad \text { et } \quad M_{1}=\left(\begin{array}{ll}
p_{1} & p_{3} \\
p_{2} & p_{0}
\end{array}\right) .
$$

Ainsi, si $\|\cdot\|$ désigne la semi norme matricielle définie par $\|A\|:=(1,0) A(1,1)^{t}$ (idée utilisée par exemple dans [10]) et $M_{I}$ la matrice $M_{\epsilon_{1}} \ldots M_{\epsilon_{n}}$, on a :

$$
\mu(I)=\left\|M_{I}\right\| .
$$

On remercie Eric Olivier [23] de nous avoir convaincu de l'intérêt de cette écriture matricielle.

Remarque. (iii) est optimale au sens où lorsque $N=1$, la mesure $\mu$ n'est en général pas quasi-Bernoulli. Par exemple, en prenant $p_{3}=0, p_{0}>p_{1}, I=0$ et $J=1 \ldots 1 \in \mathcal{F}_{n}$, d'après l'Éq. (3.13) vérifiée par $\mu$, on a :

$$
\mu(I J)=\mu(01 \ldots 1)=p_{0} \mu(J)+p_{2} \mu\left(J^{*}\right)=p_{0} \mu(1 \ldots 1)+p_{2} \mu(0 \ldots 0) .
$$

Par ailleurs, (3.14) et (3.16) entraînent que $\mu(J)=p_{1}^{n}$ et $\mu\left(J^{*}\right)=\left\|M_{0}^{n}\right\| \approx p_{0}^{n}$. Ainsi, $\mu(I J) \approx p_{0}^{n}$ et $\mu(I) \mu(J) \approx$ $p_{1}^{n}$, ce qui montre que $\mu$ n'est pas quasi-Bernoulli.

En outre, contrairement aux exemples étudiés dans [11], l'inégalité $\mu(I J) \leqslant C \mu(I) \mu(J)$ n'est ici pas vérifiée.

Venons en maintenant à la preuve de la Proposition 3.1.

Preuve de la Proposition 3.1. Tout au long de la preuve, $I=I_{\epsilon_{1} \ldots \epsilon_{n}}$ et $J=J_{\tilde{\epsilon}_{1} \ldots \tilde{\epsilon_{p}}}$ sont deux intervalles dyadiques. (i) est trivial.

Preuve de (ii). Premier cas. On suppose $N=2$.

Si $p_{2}+p_{3}=0$ alors $\mu$ est un produit de Bernoulli en base 2 (que l'on appelle aussi mesure de Besicovitch) et il est alors bien connu que $\mu(I J)=\mu(I) \mu(J)$. 
Si $p_{0}+p_{1}=0$ alors $\mu$ est un produit de Bernoulli en base 4 . En effet, dans ce cas $\mu$ vérifie

$$
\mu=p_{2} \mu \circ S_{2}^{-1}+p_{3} \mu \circ S_{3}^{-1},
$$

et on a

$$
\mu \circ S_{2}^{-1}=p_{2} \mu \circ\left(S_{2} \circ S_{2}\right)^{-1}+p_{3} \mu \circ\left(S_{2} \circ S_{3}\right)^{-1} \quad \text { et } \quad \mu \circ S_{3}^{-1}=p_{2} \mu \circ\left(S_{3} \circ S_{2}\right)^{-1}+p_{2} \mu \circ\left(S_{3} \circ S_{3}\right)^{-1} .
$$

Comme,

$$
S_{2} \circ S_{2}(x)=\frac{x}{4}+\frac{1}{4}, \quad S_{2} \circ S_{3}(x)=\frac{x}{4}, \quad S_{3} \circ S_{2}(x)=\frac{x}{4}+\frac{3}{4}, \quad S_{3} \circ S_{3}(x)=\frac{x}{4}+\frac{1}{2} ;
$$

on obtient que $\mu$ est un produit de Bernoulli en base 4. Il est alors facile de constater que $\mu$ est quasi-Bernoulli pour un codage en base 2 .

Les cas $p_{1}+p_{2}=0$ et $p_{0}+p_{3}=0$ conduisent à des études de mesures de type «Markov» (voir par exemple [4]) portées par l'intervalle $[0,1]$. Regardons par exemple le cas $p_{1}+p_{2}=0$. (L'autre situation se traite de manière similaire.) On montre facilement que :

$$
\forall n>0, \quad \frac{\mu\left(I_{\epsilon_{1} \ldots \epsilon_{n+1}}\right)}{\mu\left(I_{\epsilon_{1} \ldots \epsilon_{n}}\right)}=p_{0} \quad \text { si } \epsilon_{n}=\epsilon_{n+1} \quad \text { et } \quad \frac{\mu\left(I_{\epsilon_{1} \ldots \epsilon_{n+1}}\right)}{\mu\left(I_{\epsilon_{1} \ldots \epsilon_{n}}\right)}=p_{3} \quad \text { si } \epsilon_{n} \neq \epsilon_{n+1} .
$$

Par suite, pour connaître la masse de l'intervalle dyadique $I_{\epsilon_{1} \ldots \epsilon_{n}}$, on compte le nombre $\widetilde{N}$ de $i \in\{1, \ldots, n-1\}$ tel que $\epsilon_{i} \neq \epsilon_{i+1}$ et on trouve

$$
\mu\left(I_{\epsilon_{1} \ldots \epsilon_{n}}\right)=\tilde{p} p_{0}^{n-1-\widetilde{N}} p_{3}^{\widetilde{N}},
$$

où $\tilde{p}=p_{0}$ où $p_{3}$ selon la valeur de $\epsilon_{1}$. De cela, on déduit que $\mu$ est quasi-Bernoulli.

La terminologie Markov appliquée à ces mesures se justifie par le fait que dans ce cas $\mu$ est la loi de la variable aléatoire $X=\sum_{n=1}^{+\infty} 2^{-n} X_{n}$ où $\left(X_{n}\right)_{n} \geqslant 1$ est une chaîne de Markov homogène à états 0 et 1 , ayant pour matrice de transition

$$
\left(\begin{array}{ll}
p_{0} & p_{3} \\
p_{3} & p_{0}
\end{array}\right)
$$

et pour loi initiale $\mathbb{P}\left[X_{1}=0\right]=p_{0}$ et $\mathbb{P}\left[X_{1}=1\right]=p_{3}$.

Enfin, lorsque $p_{1}+p_{3}=0\left(\right.$ resp $\left.p_{0}+p_{2}=0\right)$, la mesure est portée par $\left[0, \frac{1}{2}\right]$ (resp. $\left.\left[\frac{1}{2}, 1\right]\right)$ et il est facile de voir qu'une propriété de type Markov est encore vérifiée dans cette situation.

Deuxième cas. On suppose $N=0$. D'après la relation (3.16), on a :

$$
\mu(I J)=\left\|M_{I J}\right\|=\left\|M_{I} M_{J}\right\| .
$$

On note $C=\max \left\{p_{0}, p_{1}, p_{2}, p_{3}\right\} / \min \left\{p_{0}, p_{1}, p_{2}, p_{3}\right\}$ et $E$ la matrice de taille 2 dont toutes les entrées de la première colonne sont égales à 1 et toutes les autres entrées sont nulles. On vérifie que pour tout $\epsilon \in\{0,1\}$, on a

$$
\frac{1}{C} E M_{\epsilon} \leqslant M_{\epsilon} \leqslant C E M_{\epsilon} \text {. }
$$

Par suite,

$$
\frac{1}{C}\left\|M_{I}\right\|\left\|M_{J}\right\|=\frac{1}{C}\left\|M_{I} E M_{J}\right\| \leqslant\left\|M_{I} M_{J}\right\| \leqslant C\left\|M_{I} E M_{J}\right\|=C\left\|M_{I}\right\|\left\|M_{J}\right\|,
$$

ce qui donne finalement

$$
\frac{1}{C} \mu(I) \mu(J) \leqslant \mu(I J) \leqslant C \mu(I) \mu(J)
$$

et termine la preuve de (ii). 
Preuve de (iii). Comme $M_{0}+M_{1}>0$, on peut trouver en raisonnant comme ci-dessus une constante $C>0$ telle que

$$
\frac{1}{C} E \leqslant M_{0}+M_{1} \quad \text { et } \quad I_{2} \leqslant C E\left(M_{0}+M_{1}\right)
$$

où $I_{2}$ est la matrice identité. Par suite,

$$
\mu\left(I \cap \sigma^{-(n+1)}(J)\right)=\mu(I 0 J)+\mu(I 1 J)=\left\|M_{I}\left(M_{0}+M_{1}\right) M_{J}\right\| \geqslant \frac{1}{C}\left\|M_{I} E M_{J}\right\|=\frac{1}{C} \mu(I) \mu(J) .
$$

De même, on a :

$$
\mu\left(I \cap \sigma^{-n}(J)\right)=\mu(I J)=\left\|M_{I} M_{J}\right\| \leqslant C\left\|M_{I} E\left(M_{0}+M_{1}\right) M_{J}\right\|=\mu(I) \mu\left(\sigma^{-1}(J)\right) .
$$

Cette relation, vraie pour tout intervalle dyadique $J$, s'étend par un argument de classe monotone au cas où $B$ est un borélien quelconque. Ainsi, en prenant $B=\sigma^{-1}(J)$, on trouve

$$
\mu\left(I \cap \sigma^{-(n+1)}(J)\right) \leqslant C \mu(I) \mu\left(\sigma^{-2}(J)\right),
$$

ce qui termine la preuve de la Proposition 3.1.

\section{Le lien avec certains graphes autoaffines}

Dans cette partie, on propose une nouvelle approche d'exemples déjà étudiés par McMullen [20], Przytycki et Urbański [26,30], Kenyon et Peres [16]. Ce point de vue est motivé par le fait que certaines mesures autosimilaires étudiées dans la partie 3 apparaissent naturellement lors de l'étude de certains graphes autoaffines.

Rappelons tout d'abord un résultat classique. Soit $0<\gamma<1$. On dit qu'une fonction $h:[0,1] \rightarrow \mathbb{R}$ est $\gamma$-holdérienne et $\gamma$-antiholdérienne si ses oscillations vérifient :

$$
\left\{\begin{array}{l}
\exists C_{1}>0, \forall x, y \in[0,1]^{2}, \quad|h(x)-h(y)| \leqslant C_{1}|x-y|^{\gamma}, \\
\exists C_{2}>0, \forall I \subset[0,1], \quad \operatorname{osc}(h, I):=\sup _{x, y \in I}|h(x)-h(y)| \geqslant C_{2}|I|^{\gamma} .
\end{array}\right.
$$

On a alors la proposition élémentaire suivante (voir [14]) :

Proposition 4.1 [14]. Soit $h$ une fonction $\gamma$-holdérienne et $\gamma$-antiholdérienne. Alors,

$$
\Delta\left(\Gamma_{h}\right)=\operatorname{Dim}\left(\Gamma_{h}\right)=2-\gamma,
$$

où $\Delta$ désigne la dimension de boîte et $\Gamma_{h}$ le graphe de $h$.

\subsection{L'exemple de McMullen}

Dans [26] Przytycki et Urbański, en modifiant légérement la construction de McMullen [20], ont mis en évidence une fonction continue $f 1 / 2$-holdérienne et $1 / 2$-antiholdérienne dont le graphe $\Gamma_{f}$ vérifie $\operatorname{dim}\left(\Gamma_{f}\right)<$ $\operatorname{Dim}\left(\Gamma_{f}\right)=3 / 2 . \Gamma_{f}$ est obtenu comme attracteur d'un système de fonctions itérées (voir figure 1). Plus précisément, pour $i=0, \ldots, 3$, on considère les affinités $A_{i}$ du carré unité $[0,1] \times[0,1]$ définies par

$$
\begin{aligned}
& A_{0}(x, y)=\left(\frac{x}{4}, \frac{y}{2}\right), \quad A_{1}(x, y)=\left(-\frac{x}{4}+\frac{1}{2}, \frac{y}{2}\right), \\
& A_{2}(x, y)=\left(\frac{x}{4}+\frac{1}{2}, \frac{y}{2}\right), \quad A_{3}(x, y)=\left(\frac{x}{4}+\frac{3}{4}, \frac{y}{2}+\frac{1}{2}\right),
\end{aligned}
$$



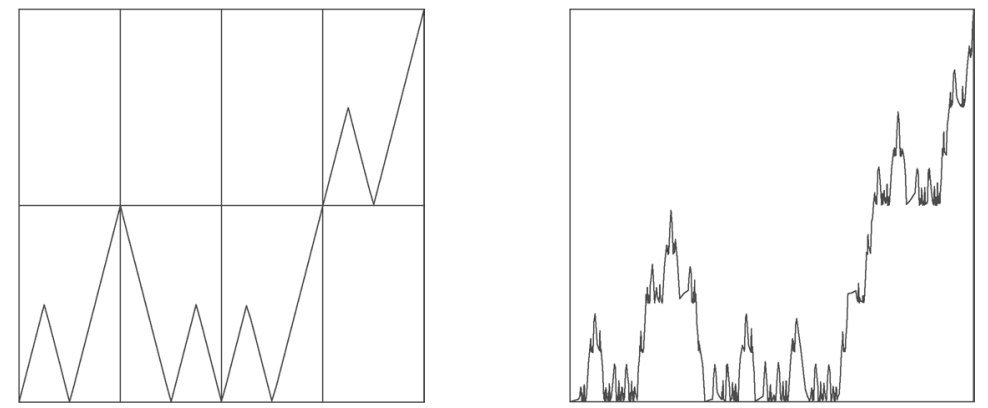

Fig. 1. Un exemple de graphe autoaffine vérifiant $\operatorname{dim}\left(\Gamma_{f}\right)<\operatorname{Dim}\left(\Gamma_{f}\right)$.

et on appelle $\Gamma_{f}$ l'unique ensemble compact vérifiant

$$
\Gamma_{f}=\bigcup_{i=0}^{3} A_{i}\left(\Gamma_{f}\right)
$$

Pour estimer $\operatorname{dim}\left(\Gamma_{f}\right)$, on introduit la famille de mesures autoaffines, portées par $\Gamma_{f}$, qui vérifient

$$
v=\sum_{i=0}^{3} q_{i} \nu \circ A_{i}^{-1}
$$

et on cherche à en calculer la dimension. Pour cela essayons de voir comment s'effectue le calcul de $\tau_{v}$.

On note $\mathcal{G}_{n}$ la famille des carrés 4 -adiques de nième génération inclus dans le carré unité. En d'autres termes,

$$
\mathcal{G}_{n}=\left\{I=\left[k_{1} / 4^{n},\left(k_{1}+1\right) / 4^{n}\left[\times\left[k_{2} / 4^{n},\left(k_{2}+1\right) / 4^{n}\left[, 0 \leqslant k_{1}<4^{n}, 0 \leqslant k_{2}<4^{n}\right\} .\right.\right.\right.\right.
$$

Par ailleurs, on note $\mathcal{R}_{n}$ l'ensemble des rectangles suivants :

$$
\mathcal{R}_{n}=\left\{R=\left[k_{1} / 4^{n},\left(k_{1}+1\right) / 4^{n}\left[\times\left[k_{2} / 2^{n},\left(k_{2}+1\right) / 2^{n}\left[, 0 \leqslant k_{1}<4^{n}, 0 \leqslant k_{2}<2^{n}\right\} .\right.\right.\right.\right.
$$

Enfin, on note $\mathcal{F}_{n}$ (resp. $\mathcal{P}_{n}$ ) l'ensemble des intervalles dyadiques (resp. 4-adiques) de nième génération inclus dans l'intervalle $[0,1]$. Autrement dit,

$$
\mathcal{F}_{n}=\left\{J=\left[k / 2^{n},(k+1) / 2^{n}\left[, 0 \leqslant k<2^{n}\right\}\right.\right.
$$

et

$$
\mathcal{P}_{n}=\left\{K=\left[k / 4^{n},(k+1) / 4^{n}\left[, 0 \leqslant k<4^{n}\right\} .\right.\right.
$$

Par définition,

$$
\tau_{n}(q)=\frac{1}{n \log 4} \log \left(\sum_{I \in \mathcal{G}_{n}} v(I)^{q}\right) .
$$

En effectuant une sommation par paquets on obtient

$$
\sum_{I \in \mathcal{G}_{n}} v(I)^{q}=\sum_{R \in \mathcal{R}_{n}} \sum_{I \in \mathcal{G}_{n}, I \subset R} v(I)^{q}=\sum_{R \in \mathcal{R}_{n}} \sum_{I \in \mathcal{G}_{n}, I \subset R} v(R)^{q}\left(\frac{v(I)}{v(R)}\right)^{q} .
$$

A $R$ fixé, regardons maintenant comment on peut déterminer

$$
S=\sum_{I \in \mathcal{G}_{n}, I \subset R}\left(\frac{v(I)}{v(R)}\right)^{q} .
$$


On remarque que l'ensemble des carrés $I \in \mathcal{G}_{n}$ inclus dans le rectangle $R$ découpe ce dernier en $2^{n}$ tranches horizontales. En outre, la mesure autoaffine $v$ est répartie de la même manière dans $R$ que dans le carré unité. Ainsi, en voyant $R$ comme un zoom du carré unité par une affinité, on déduit que

$$
S=\sum_{J \in \mathcal{F}_{n}} v_{y}(J)^{q},
$$

où $v_{y}$ est la mesure projetée de $v$ sur l'axe des $y$. Par ailleurs, avec des notations évidentes, on a facilement :

$$
\sum_{R \in \mathcal{R}_{n}} v(R)^{q}=\sum_{K \in \mathcal{P}_{n}} v_{x}(K)^{q} .
$$

Par suite, la relation (4.2) permet dégager la proposition suivante :

\section{Proposition 4.2.}

$$
\tau_{v}=\tau_{v_{x}}+\frac{1}{2} \tau_{v_{y}}
$$

On observe tout d'abord que le calcul de $\tau_{v_{x}}$ s'apparente au calcul de la fonction $\tau$ d'un produit de Bernoulli en base 4 de paramètre $q_{0}, q_{1}, q_{2}, q_{3}$. En outre, dans cette situation, $v_{y}$ est un produit de Bernoulli en base 2 . En effet, on a

$$
v_{y}=v \circ \pi_{y}^{-1}=\left(\sum q_{i} v \circ A_{i}^{-1}\right) \circ \pi_{y}^{-1}=\sum q_{i} \nu \circ\left(\pi_{y} \circ A_{i}\right)^{-1} .
$$

De plus, si on définit $S_{0}$ et $S_{1}$ par

$$
S_{0}(x)=\frac{x}{2} \quad \text { et } \quad S_{1}(x)=\frac{x}{2}+\frac{1}{2}
$$

alors

$$
\pi_{y} \circ A_{0}=\pi_{y} \circ A_{1}=\pi_{y} \circ A_{2}=S_{0} \circ \pi_{y} \quad \text { et } \quad \pi_{y} \circ A_{3}=S_{1} \circ \pi_{y} .
$$

Ainsi, $v_{y}$ vérifie

$$
v_{y}=\left(q_{0}+q_{1}+q_{2}\right) \nu \circ\left(S_{0} \circ \pi_{y}\right)^{-1}+q_{3} v \circ\left(S_{1} \circ \pi_{y}\right)^{-1}=\left(q_{0}+q_{1}+q_{2}\right) v_{y} \circ S_{0}^{-1}+q_{3} v_{y} \circ S_{1}^{-1} .
$$

Par suite, $v_{y}$ est un produit de Bernoulli de paramètre $q_{0}+q_{1}+q_{2}$ et le calcul de $\tau_{v_{y}}$ est alors classique.

La relation (4.3) et le fait que $\tau_{v_{x}}$ et $\tau_{v_{y}}$ soient dérivables en 1 nous assurent en particulier :

Théorème 4.1. Pour tout choix des coefficients $q_{0}, q_{1}, q_{2}, q_{3}$; la mesure v est unidimensionnelle et vérifie :

$$
\operatorname{dim}(v)=-\tau_{v}^{\prime}(1)=-\tau_{v_{x}}^{\prime}(1)-\frac{1}{2} \tau_{v_{y}}^{\prime}(1)
$$

La dérivabilité de la fonction $\tau$ au point 1 va nous permettre de retrouver la minoration de la dimension de $\Gamma_{f}$ que McMullen avait obtenue en utilisant la loi forte des grands nombres. En effet, le Théorème 4.1 et l'analyse des mesures projetées $v_{x}$ et $v_{y}$ montrent que :

$$
\begin{aligned}
\operatorname{dim}(v) & =-\frac{1}{\log 4}\left(\left(q_{0}+q_{1}+q_{2}\right) \log \left(q_{0}+q_{1}+q_{2}\right)+q_{0} \log q_{0}+q_{1} \log q_{1}+q_{2} \log q_{2}+2 q_{3} \log q_{3}\right) \\
& :=\theta\left(q_{0}, q_{1}, q_{2}, q_{3}\right) .
\end{aligned}
$$


On trouve facilement que $\theta$ est maximale pour $q_{0}=q_{1}=q_{2}=\frac{1}{\sqrt{3}(1+\sqrt{3})}$ et $q_{3}=\frac{1}{1+\sqrt{3}}$. De plus, son maximum vaut $\frac{1}{\log 2} \log (1+\sqrt{3})$. Par suite,

$$
\operatorname{dim}\left(\Gamma_{f}\right) \geqslant \operatorname{dim}(v) \geqslant \frac{1}{\log 2} \log (1+\sqrt{3}) .
$$

En outre, McMullen a montré que pour cette valeur particulière de $\left(q_{0}, q_{1}, q_{2}, q_{3}\right)$ la mesure $v$ vérifie

$$
\forall x \in \Gamma_{f}, \quad \liminf _{n \rightarrow \infty} \frac{\log v\left(I_{n}(x)\right)}{\log \left|I_{n}(x)\right|} \leqslant \frac{1}{\log 2} \log (1+\sqrt{3}),
$$

ce qui donne finalement de manière classique (voir par exemple [6]) :

$$
\operatorname{dim}\left(\Gamma_{f}\right)=\frac{1}{\log 2} \log (1+\sqrt{3})<\frac{3}{2} .
$$

\subsection{L'exemple d'Urbański}

Dans [30] Urbański modifie à nouveau la construction de McMullen en prenant

$$
\begin{aligned}
& A_{0}(x, y)=\left(\frac{x}{4}, \frac{y}{2}\right), \quad A_{1}(x, y)=\left(\frac{x}{4}+\frac{1}{4},-\frac{y}{2}+\frac{1}{2}\right), \\
& A_{2}(x, y)=\left(\frac{x}{4}+\frac{1}{2}, \frac{y}{2}\right), \quad A_{3}(x, y)=\left(\frac{x}{4}+\frac{3}{4}, \frac{y}{2}+\frac{1}{2}\right) .
\end{aligned}
$$

L'attracteur est encore le graphe d'une fonction continue $f$ 1/2-holdérienne et 1/2-antiholdérienne (voir Fig. 2), mais dans cette situation Urbański a montré que :

$$
\operatorname{dim}\left(\Gamma_{f}\right)=\operatorname{Dim}\left(\Gamma_{f}\right)=\frac{3}{2} .
$$

Voyons comment on peut utiliser les résultats de la partie 3 pour traiter cet exemple et retrouver ainsi simplement le résultat annoncé.

Dans ce cas, le calcul de la fonction $\tau$ de la mesure autoaffine $v$ vérifiant l'Éq. (4.1) est différent. En effet, si

$$
S_{0}(x)=\frac{x}{2}, \quad S_{1}(x)=\frac{x}{2}+\frac{1}{2} \quad \text { et } \quad S_{2}(x)=-\frac{x}{2}+\frac{1}{2}
$$

alors

$$
\pi_{y} \circ A_{0}=\pi_{y} \circ A_{2}=S_{0} \circ \pi_{y}, \quad \pi_{y} \circ A_{3}=S_{1} \circ \pi_{y} \quad \text { et } \quad \pi_{y} \circ A_{1}=S_{2} \circ \pi_{y} .
$$
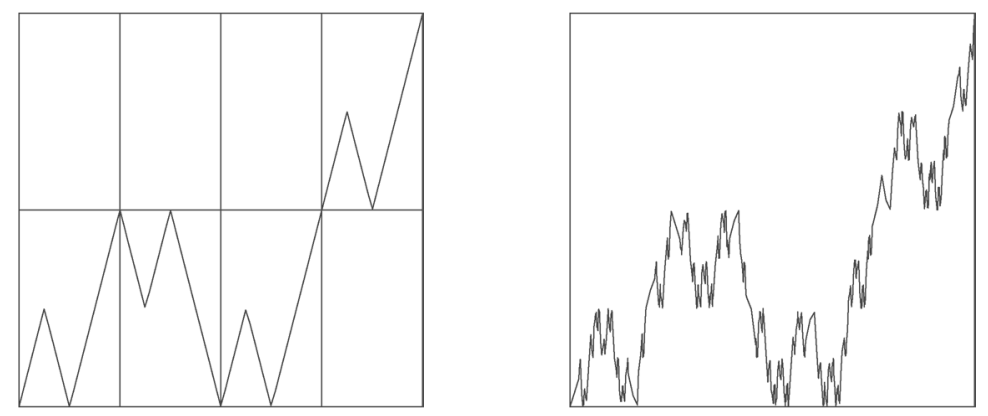

Fig. 2. Un exemple de graphe autoaffine vérifiant $\operatorname{dim}\left(\Gamma_{f}\right)=\operatorname{Dim}\left(\Gamma_{f}\right)$. 
Par suite, la mesure projetée $v_{y}$ vérifie

$$
v_{y}=\left(q_{0}+q_{2}\right) v_{y} \circ S_{0}^{-1}+q_{3} v_{y} \circ S_{1}^{-1}+q_{1} v_{y} \circ S_{2}^{-1},
$$

et apparaît comme un cas particulier des mesures étudiées dans la partie 3.6. Ainsi, le Théorème 3.2 assure entre autres qu'elle est unidimensionnelle et que l'on a :

$$
\operatorname{dim}\left(v_{y}\right)=-\tau_{v_{y}}^{\prime}(1)
$$

On note que la mesure $v_{y}$ entre dans la situation (iii) de la Proposition 3.1 et n'est donc pas en général quasiBernoulli.

Les remarques précédentes et la relation (4.3) qui reste vraie dans cette situation nous permettent ainsi de relier à nouveau la dimension de $v$ avec la dimension de ses mesures projetées à travers l'égalité :

$$
\operatorname{dim}(v)=\operatorname{dim}\left(v_{x}\right)+\frac{1}{2} \operatorname{dim}\left(v_{y}\right) .
$$

Toutefois, dans ce cas de figure, la présence d'overlaps rend le calcul de $\tau_{v_{y}}^{\prime}(1)$ difficile. Cependant, on obtient facilement la proposition suivante :

Proposition 4.3. Soit $\mathrm{d} x$ la mesure de Lebesgue sur l'intervalle [0, 1].

Si $q_{0}=q_{1}=q_{2}=q_{3}=\frac{1}{4}$ alors $v_{y}=2(1-x) \mathrm{d} x$.

Comme ici $v_{x}=\mathrm{d} x$, la relation (4.5) et la Proposition 4.3 entraînent que

$$
\operatorname{dim}\left(\Gamma_{f}\right) \geqslant \operatorname{dim}(v)=\operatorname{dim}\left(v_{x}\right)+\frac{1}{2} \operatorname{dim}\left(v_{y}\right)=1+\frac{1}{2} 1=\frac{3}{2},
$$

et permettent de retrouver facilement le résultat de Urbański :

\section{Corollaire 4.1.}

$$
\operatorname{dim}\left(\Gamma_{f}\right)=\frac{3}{2} .
$$

Remarque. Plus généralement, si

$$
\mu=\sum_{i=0}^{3} p_{i} \mu \circ S_{i}^{-1},
$$

avec

$$
S_{0}(x)=\frac{x}{2}, \quad S_{1}(x)=\frac{x}{2}+\frac{1}{2}, \quad S_{2}(x)=-\frac{x}{2}+\frac{1}{2}, \quad S_{3}(x)=-\frac{x}{2}+1 ;
$$

on montre dans [28] que $\operatorname{dim}(\mu)=1$ équivaut à $\mu$ absolument continue par rapport $\mathrm{d} x$, ce qui équivaut encore à $p_{0}+p_{3}=0.5$ ou $p_{0}+p_{2}=0.5$.

Montrons à présent la Proposition 4.3.

Preuve de la Proposition 4.3. On note $\phi(x)=2(1-x)$ et $m$ la mesure $m=\phi \mathrm{d} x$. Pour établir la proposition, on va montrer que $m$ satisfait l'équation fonctionnelle vérifiée par $v_{y}$, à savoir

$$
m=\frac{1}{2} m \circ S_{0}^{-1}+\frac{1}{4} m \circ S_{1}^{-1}+\frac{1}{4} m \circ S_{2}^{-1} .
$$

En effet, pour toute fonction mesurable $\psi \geqslant 0$ sur l'intervalle [0, 1], on a : 


$$
\begin{aligned}
I(\psi) & :=\frac{1}{2} \int_{[0,1]} \psi \mathrm{d}\left(m \circ S_{0}^{-1}\right)+\frac{1}{4} \int_{[0,1]} \psi \mathrm{d}\left(m \circ S_{1}^{-1}\right)+\frac{1}{4} \int_{[0,1]} \psi \mathrm{d}\left(m \circ S_{2}^{-1}\right) \\
& =\int_{[0,1]}\left(\psi\left(\frac{x}{2}\right)+\frac{1}{2} \psi\left(\frac{x}{2}+\frac{1}{2}\right)+\frac{1}{2} \psi\left(-\frac{x}{2}+\frac{1}{2}\right)\right)(1-x) \mathrm{d} x .
\end{aligned}
$$

Par des formules de changements de variables, on obtient

$$
\begin{aligned}
I(\psi) & =2 \int_{[0,1 / 2]} \psi(x)(1-2 x) \mathrm{d} x+\int_{[1 / 2,1]} \psi(x)(2-2 x) \mathrm{d} x+\int_{[0,1 / 2]} \psi(x) 2 x \mathrm{~d} x \\
& =\int_{[0,1 / 2]} \psi(x)(2-2 x) \mathrm{d} x+\int_{[1 / 2,1]} \psi(x)(2-2 x) \mathrm{d} x=\int_{[0,1]} \psi(x) \mathrm{d} m,
\end{aligned}
$$

ce qui prouve la Proposition 4.3.

\subsection{L'exemple de Kenyon et Peres}

Terminons ce travail en disant quelques mots sur le lien entre l'exemple étudié par Kenyon et Peres dans [16] et la mesure $\mu$ de la partie 3.4 associée à $b=1$. En considérant les affinités

$$
A_{0}(x, y)=\left(\frac{x}{3}, \frac{y}{2}\right), \quad A_{1}(x, y)=\left(\frac{x}{3}+\frac{1}{3}, \frac{y}{2}+\frac{1}{4}\right), \quad A_{2}(x, y)=\left(\frac{x}{3}+\frac{2}{3}, \frac{y}{2}+\frac{1}{2}\right) ;
$$

Kenyon et Peres ont mis en évidence un ensemble autoaffine (voir Fig. 3) dont la dimension de Hausdorff ne peut pas être calculée à l'aide d'une mesure autoaffine. Plus précisément, si

$$
E=\bigcup_{i=0}^{2} A_{i}(E)
$$

et si on introduit la famille de mesures autoaffines vérifiant

$$
v=p_{0} v \circ A_{0}^{-1}+p_{1} v \circ A_{1}^{-1}+p_{2} v \circ A_{2}^{-1},
$$

alors pour tout choix de $p_{0}, p_{1}, p_{2}$; on a :

$$
\operatorname{dim}(v)=\operatorname{dim}\left(v_{x}\right)+\left(1-\frac{\log 2}{\log 3}\right) \operatorname{dim}\left(v_{y}\right)<\operatorname{dim}(E) .
$$
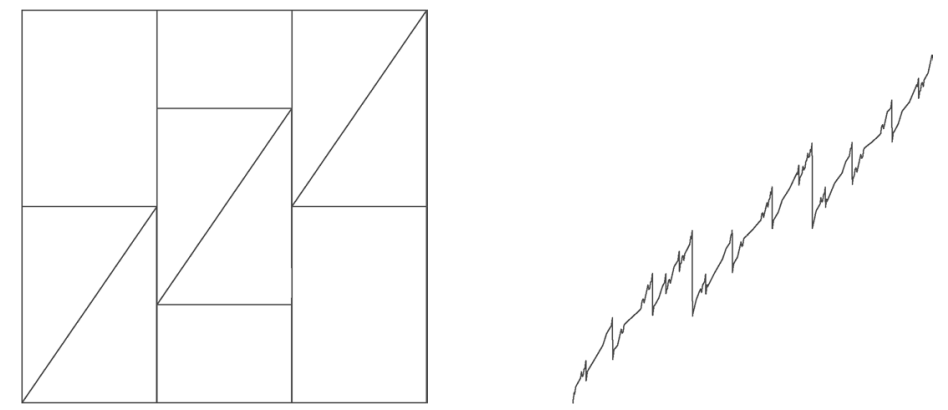

Fig. 3. Un exemple de graphe autoaffine dont la dimension ne peut pas être calculée à l'aide d'une mesure autoaffine. 
Kenyon et Peres obtiennent les inégalités $\operatorname{dim}(v)<\operatorname{dim}(E)<\operatorname{Dim}(E)$ grâce à des calculs approchés de $\operatorname{dim}(v)$ et $\operatorname{dim}(E)$.

Voyons comment l'égalité $\operatorname{dim}(v)=\operatorname{dim}\left(v_{x}\right)+(1-\log 2 / \log 3) \operatorname{dim}\left(v_{y}\right)$ peut être simplement justifiée. Un calcul similaire à celui effectué dans la partie 4.1 nous fournit :

$$
\tau_{v}=\tau_{v_{x}}+\left(1-\frac{\log 2}{\log 3}\right) \tau_{v_{y}}
$$

Ici, $v_{x}$ est encore un produit de Bernoulli tandis que $v_{y}$ est la mesure $\mu$ étudiée dans la partie 3.4 en prenant $b=1$. Ainsi, les Théorèmes 3.1 et 2.2 assurent l'existence $\tau_{v_{y}}^{\prime}(1)$. Par suite, $v$ est unidimensionnelle et on a bien l'égalité annoncée.

Cependant, comme pour l'exemple d'Urbański la présence d'overlaps rend le calcul de de la dimension de $v_{y}$ difficile. Toutefois, on peut obtenir une approximation numérique de cette valeur à l'aide d'une formule d'entropie. En effet, comme $\tau_{v_{y}}$ est dérivable au point 1, on a (voir [13]) :

$$
\operatorname{dim}\left(v_{y}\right)=\operatorname{Dim}\left(v_{y}\right)=\lim _{n \rightarrow \infty}-\frac{1}{n \log 2} \sum_{J \in \mathcal{F}_{n}} v_{y}(J) \log v_{y}(J) .
$$

En outre, on a vu dans la partie 3.4 que le calcul des quantités $v_{y}(J)$ peut s'effectuer à l'aide d'un produit de matrices.

Regardons à présent comment on peut obtenir de façon élémentaire un encadrement $\operatorname{de} \operatorname{dim}(E)$. Pour $0<p<\frac{1}{2}$, on considère la mesure autoaffine $v_{p}$ portée par $E$ vérifiant :

$$
v_{p}=p v_{p} \circ A_{0}^{-1}+(1-2 p) v_{p} \circ A_{1}^{-1}+p v_{p} \circ A_{2}^{-1} .
$$

En vertu de ce qui précède, on a :

$$
\operatorname{dim}\left(v_{p}\right)=\operatorname{dim}\left(\left(v_{p}\right)_{x}\right)+\left(1-\frac{\log 2}{\log 3}\right) \operatorname{dim}\left(\left(v_{p}\right)_{y}\right) .
$$

On va pouvoir obtenir une minoration $\operatorname{de} \operatorname{dim}(E)$ grâce à la proposition suivante :

Proposition 4.4. Pour tout $0<p<\frac{1}{2}$, on a la minoration

$$
\operatorname{dim}\left(\left(v_{p}\right)_{y}\right) \geqslant g(p)
$$

où

$$
g(p)=\max \left(-\frac{1}{\log 2}(p \log p+(1-p) \log (1-p)),-\frac{1}{\log 2}(2 p \log 2 p+(1-2 p) \log (1-2 p))\right) .
$$

En utilisant la Proposition 4.4 et la relation (4.6), on trouve donc

$$
\operatorname{dim}(E) \geqslant \operatorname{dim}\left(\left(v_{p}\right)_{x}\right)+\left(1-\frac{\log 2}{\log 3}\right) g(p):=f(p) .
$$

Par ailleurs, $\left(v_{p}\right)_{x}$ est un produit de Bernoulli en base 3 dont la dimension vaut

$$
\operatorname{dim}\left(\left(v_{p}\right)_{x}\right)=-\frac{1}{\log 3}(2 p \log p+(1-2 p) \log (1-2 p)) .
$$

De plus, en étudiant les variations de $f$ sur $\left[0, \frac{1}{2}\right]$, on trouve qu'elle est maximale en $p=2^{\alpha} /\left(2\left(1+2^{\alpha}\right)\right)$ où $\alpha=\log 2 / \log 3$ et ce maximum vaut $(1 / \log 2) \log \left(1+2^{\alpha}\right)$. On obtient finalement le résultat suivant : 
Théorème 4.2. Soit $\alpha=\log 2 / \log 3$. On $a$ :

$$
1.349 \approx \frac{1}{\log 2} \log \left(1+2^{\alpha}\right) \leqslant \operatorname{dim}(E) \leqslant 2-\alpha \approx 1.369 .
$$

Remarque. Kenyon et Peres ont en fait obtenu que $\operatorname{dim}(E) \approx 1.366$. Par ailleurs, la borne inférieure obtenue ici correspond à la dimension de Hausdorff de l'ensemble autoaffine étudié par McMullen en prenant pour affinités du carré unité :

$$
A_{0}(x, y)=\left(\frac{x}{3}, \frac{y}{2}\right), \quad A_{1}(x, y)=\left(\frac{x}{3}+\frac{1}{3}, \frac{y}{2}+\frac{1}{2}\right), \quad A_{2}(x, y)=\left(\frac{x}{3}+\frac{2}{3}, \frac{y}{2}\right) .
$$

Pour terminer ce travail, établissons la Proposition 4.4.

Preuve de la Proposition 4.4. Montrons tout d'abord que :

$$
\operatorname{dim}\left(\left(v_{p}\right)_{y}\right) \geqslant-\frac{1}{\log 2}(p \log p+(1-p) \log (1-p)) .
$$

Cela provient du résultat suivant démontré par Heurteaux dans [12,13].

Lemme 4.1 [12,13]. Soient un réel $0.5 \leqslant \beta<1$ et $m$ une mesure de probabilité sur $[0,1$ [ tels que pour tout intervalle dyadique I, on ait :

$$
\frac{m(I 0)}{m(I)} \leqslant \beta \quad \text { et } \quad \frac{m(I 1)}{m(I)} \leqslant \beta
$$

Alors,

$$
\operatorname{dim}_{*}(m) \geqslant-\frac{1}{\log 2}(\beta \log \beta+(1-\beta) \log (1-\beta)) .
$$

Ici, on va montrer que $\left(v_{p}\right)_{y} \circ \sigma^{-1}$ vérifie (4.8) pour $\beta=1-p$. Les résultats de la partie 3 donnent alors :

$$
\operatorname{dim}\left(\left(v_{p}\right)_{y}\right)=-\tau_{\left(v_{p}\right)_{y}}^{\prime}(1)=-\tau_{\left(v_{p}\right)_{y} \circ \sigma^{-1}}^{\prime}(1)=\operatorname{dim}\left(\left(v_{p}\right)_{y} \circ \sigma^{-1}\right) \geqslant-\frac{1}{\log 2}(p \log p+(1-p) \log (1-p)) .
$$

De plus, on trouve par un calcul élémentaire que les matrices $M_{0}$ et $M_{1}$ associées à $\left(v_{p}\right)_{y}$ s'écrivent

$$
M_{0}=\left(\begin{array}{cc}
p & 0 \\
p & 1-2 p
\end{array}\right), \quad M_{1}=\left(\begin{array}{cc}
1-2 p & p \\
0 & p
\end{array}\right) .
$$

Ainsi, pour tout intervalle dyadique $I$,

$$
\left(v_{p}\right)_{y} \circ \sigma^{-1}(I)=\left(v_{p}\right)_{y}(0 I)+\left(v_{p}\right)_{y}(1 I)=\frac{\left[\left(\left(v_{p}\right)_{y}\right)_{0}+\left(\left(v_{p}\right)_{y}\right)_{1}\right](I)}{2}=\frac{\left\|M_{I}\right\|}{2},
$$

où on a noté $\left\|M_{I}\right\|$ la somme des coefficients de $M_{I}$. Par suite, pour tout $\epsilon \in\{0,1\}$, on a

$$
\frac{\left(v_{p}\right)_{y} \circ \sigma^{-1}(I \epsilon)}{\left(v_{p}\right)_{y} \circ \sigma^{-1}(I)}=\frac{\left\|M_{I} M_{\epsilon}\right\|}{\left\|M_{I}\right\|} \leqslant 1-p,
$$

ce qui établit la relation (4.8) pour $\left(v_{p}\right)_{y} \circ \sigma^{-1}$ et montre (4.7).

Pour terminer, montrons maintenant que :

$$
\operatorname{dim}\left(\left(v_{p}\right)_{y}\right) \geqslant-\frac{1}{\log 2}(2 p \log 2 p+(1-2 p) \log (1-2 p)) .
$$

Cela provient du résultat suivant : 
Lemme 4.2. Soient un réel $0.5 \leqslant \beta<1$ et $m$ une mesure de probabilité $\sigma$ invariante sur $[0,1$ [ tels que pour tout intervalle dyadique I, on ait :

$$
\frac{m(0 I)}{m(I)} \leqslant \beta \quad \text { et } \quad \frac{m(1 I)}{m(I)} \leqslant \beta .
$$

Alors,

$$
\operatorname{dim}_{*}(m) \geqslant-\frac{1}{\log 2}(\beta \log \beta+(1-\beta) \log (1-\beta)) .
$$

On montre que $\left(v_{p}\right)_{y} \circ \sigma^{-1}$ vérifie (4.10) pour $\beta=\max (2 p, 1-2 p)$ en utilisant des arguments similaires à ceux qui ont permis d'établir (4.8) pour cette même mesure. Pour obtenir la relation (4.9), il reste donc à vérifier que $\left(v_{p}\right)_{y} \circ \sigma^{-2}=\left(v_{p}\right)_{y} \circ \sigma^{-1}$. Cette égalité est la conséquence d'un calcul simple utilisant l'equation fonctionnelle vérifiée par $\left(v_{p}\right)_{y}$.

Pour achever la preuve de la Proposition 4.4, il reste à établir le Lemme 4.2.

Preuve du Lemme 4.2. Pour $q \geqslant 1$ et pour tout entier $n$ positif, on a :

$$
\sum_{I \in \mathcal{F}_{n}} m(I)^{q}=\sum_{I \in \mathcal{F}_{n-1}}\left[\left(\frac{m(0 I)}{m(I)}\right)^{q}+\left(\frac{m(1 I)}{m(I)}\right)^{q}\right] m(I)^{q} .
$$

La $\sigma$ invariance de $m$ nous indique que $\frac{m(0 I)}{m(I)}+\frac{m(1 I)}{m(I)}=1$. Les variations de la fonction $x \rightarrow x^{q}+(1-x)^{q}$ nous montrent alors que

$$
\forall n, \quad \sum_{I \in \mathcal{F}_{n}} m(I)^{q} \leqslant\left[\beta^{q}+(1-\beta)^{q}\right] \sum_{I \in \mathcal{F}_{n-1}} m(I)^{q} .
$$

En itérant cette inégalité, on obtient

$$
\forall q \geqslant 1, \quad \tau(q) \leqslant \frac{1}{\log 2} \log \left(\beta^{q}+(1-\beta)^{q}\right),
$$

ce qui fournit de manière classique (voir par exemple [13]) la minoration

$$
\operatorname{dim}_{*}(m) \geqslant-\frac{1}{\log 2}(\beta \log \beta+(1-\beta) \log (1-\beta))
$$

et termine la preuve du Lemme 4.2.

Remarque finale. On peut de la même manière améliorer sensiblement l'encadrement fourni en étudiant les quotients

$$
\frac{\left(v_{p}\right)_{y} \circ \sigma^{-1}(I K)}{\left(v_{p}\right)_{y} \circ \sigma^{-1}(I)} \text { et } \frac{\left(v_{p}\right)_{y} \circ \sigma^{-1}(K I)}{\left(v_{p}\right)_{y} \circ \sigma^{-1}(I)}
$$

pour des intervalles dyadiques $K$ appartenant à une génération donnée bien choisie.

\section{Remerciements}

L'auteur remercie Yanick Heurteaux pour l'attention qu'il a portée à ce travail. 


\section{Références}

[1] P. Billingsley, Ergodic Theory and Information, Wiley, New York, 1965.

[2] F. BenNasr, Analyse multifractale de mesures, C. R. Acad. Sci. Paris Sér. I Math. 319 (1994) 807-810.

[3] F. BenNasr, I. Bhouri, Spectre multifractal de mesures boréliennes sur $\mathbb{R}^{d}$, C. R. Acad. Sci. Paris Sér. I Math. 325 (1997) $253-256$.

[4] F. BenNasr, I. Bhouri, Y. Heurteaux, The validity of the multifractal formalism: results and examples, Adv. in Math. 165 (2002) $264-284$.

[5] G. Brown, G. Michon, J. Peyrière, On the multifractal analysis of measures, J. Statist. Phys. 66 (1992) 775-790.

[6] K. Falconer, Fractal Geometry, Mathematical Foundations and Applications, Wiley, New York, 1990.

[7] K. Falconer, Techniques in Fractal Geometry, Wiley, New York, 1997.

[8] A.H. Fan, Sur la dimension inférieure des mesures, Studia Math. 111 (1994) 1-17.

[9] D.J. Feng, The smoothness of $L^{q}$-spectrum of self-similar measures with overlaps, J. London Math. Soc. 68 (2003) $102-118$.

[10] D.J. Feng, K.S. Lau, The pressure function for products of non-negative matrices, Math. Res. Lett. 9 (2002) $363-378$.

[11] D.J. Feng, K.S. Lau, Differentiability of pressure functions for products of non-negative matrices, preprint.

[12] Y. Heurteaux, Sur la comparaison des mesures avec les mesures de Hausdorff, C. R Acad. Sci. Paris Sér. I Math. 321 (1995) 61-65.

[13] Y. Heurteaux, Estimations de la dimension inférieure et de la dimension supérieure des mesures, Ann. Inst. H. Poincaré Probab. Statist. 34 (1998) 309-338.

[14] Y. Heurteaux, Weierstrass function with random phases, Trans. Amer. Math. Soc. 335 (2003) 3065-3077.

[15] J.E. Hutchinson, Fractals and self similarity, Indiana Univ. Math. J. 30 (1981) 713-747.

[16] R. Kenyon, Y. Peres, Hausdorff dimension of Sofic affine-invariant sets, Israel J. Math. 94 (1996) 127-138.

[17] K.S. Lau, S.M. Ngai, $L^{q}$-spectrum of the Bernoulli convolution associated with the golden ratio, Studia Math. 131 (1998) $225-251$.

[18] K.S. Lau, S.M. Ngai, Multifractal measures and a weak separation condition, Adv. in Math. 141 (1999) 45-96.

[19] G. Michon, Mesures de Gibbs sur les Cantor réguliers, Ann. Inst. H. Poincaré Phys. Théor. 58 (1983) 267-285.

[20] C. McMullen, The Hausdorff dimension of general Sierpiński carpets, Nagoya Math. J. 96 (1984) 1-9.

[21] S.M. Ngai, A dimension result arising from the $L^{q}$-spectrum of a measure, Proc. Amer. Math. Soc. 125 (1997) $2943-2951$.

[22] S.M. Ngai, Y. Wang, Hausdorff dimension of self-similar sets with overlaps, J. London Math. Soc. 63 (2001) 655-672.

[23] E. Olivier, Communication privée.

[24] L. Olsen, A multifractal formalism, Adv. in Math. 116 (1995) 82-196.

[25] J. Peyrière, Multifractal measures, in: Proc. NATO Adv. Study Inst. Il Ciocco, vol. 372, 1997, pp. 175-186.

[26] F. Przytycki, M. Urbański, On Hausdorff dimension of some fractal sets, Studia Math. 93 (1989) 155-186.

[27] M. Tamashiro, Dimensions in a separable metric space, Kyushu J. Math. 49 (1995) 143-162.

[28] B. Testud, Thèse de doctorat, Université Blaise Pascal, Clermont-Ferrand, 2004.

[29] C. Tricot Jr, Two definitions of fractional dimension, Math. Proc. Cambridge Philos. Soc. 91 (1982) 57-74.

[30] M. Urbański, The Hausdorff dimension of the graphs of continuous self-affine functions, Proc. Amer. Math. Soc. 108 (1990) $921-930$. 